MAD-TH-07-05

CERN-PH-TH/2007-070

\title{
Black Holes and Large Order Quantum Geometry
}

\author{
Min-xin Huang ${ }^{a}$, Albrecht Klemm ${ }^{a, b}$, Marcos Mariño $^{c}$ and Alireza Tavanfar ${ }^{c, d}$ \\ ${ }^{a}$ Department of Physics and ${ }^{b}$ Department of Mathematics, \\ University of Wisconsin, Madison, WI 53706, USA \\ ${ }^{c}$ Department of Physics, CERN \\ Geneva 23, CH-1211 Switzerland \\ ${ }^{d}$ Institute for Studies in Theoretical Physics and Mathematics (IPM) \\ P.O. Box 19395-5531, Tehran, Iran

\begin{abstract}
We study five-dimensional black holes obtained by compactifying M theory on CalabiYau threefolds. Recent progress in solving topological string theory on compact, oneparameter models allows us to test numerically various conjectures about these black holes. We give convincing evidence that a microscopic description based on GopakumarVafa invariants accounts correctly for their macroscopic entropy, and we check that highly nontrivial cancellations - which seem necessary to resolve the so-called entropy enigma in the OSV conjecture- do in fact occur. We also study analytically small $5 \mathrm{~d}$ black holes obtained by wrapping M2 branes in the fiber of K3 fibrations. By using heterotic/type II duality we obtain exact formulae for the microscopic degeneracies in various geometries, and we compute their asymptotic expansion for large charges.
\end{abstract}

April 2007

minxin@physics.wisc.edu, aklemm@physics.wisc.edu, marcos@mail.cern.ch, alireza.tavanfar@cern.ch 


\section{Contents}

1 Introduction $\quad 1$

2 Microscopic and macroscopic counting for 5 d black holes 4

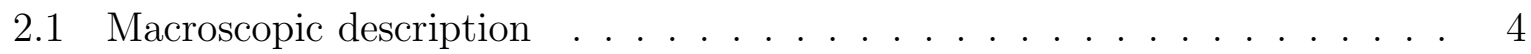

2.2 Microscopic description . . . . . . . . . . . . . . . . . 6

3 One-parameter models

3.1 Topological strings on one-parameter models . . . . . . . . . . . . . 8

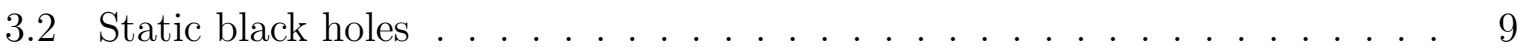

3.3 Spinning black holes . . . . . . . . . . . . . . . . . 15

4 Asymptotics of the Donaldson-Thomas invariants

5 K3 fibrations $\quad 22$

5.1 Topological strings on K3 fibrations . . . . . . . . . . . . . . 22

5.2 Microscopic degeneracies and their asymptotic expansion . . . . . . . . . 25

5.3 Macroscopic entropy for small black holes . . . . . . . . . . . . . . . . . 31

6 Conclusions

A General features of the instanton expansion 34

\section{Introduction}

String theory can provide in many situations a precise microscopic description of supersymmetric black holes which reproduces for large charges the Bekenstein-Hawking entropy [39]. Degeneracies of microstates that are highly protected by supersymmetry are often counted by mathematically well understood quasitopological quantities related to the compactification manifold. For example, the computation of microstates of the D1-D5 system is encoded in the elliptic genus of a symmetric product of a hyperKähler manifold (see [14, 11] for a review of these computations).

A very challenging class of black holes in string theory is obtained by compactifying M theory on a Calabi-Yau manifold $X$ with generic $S U(3)$ holonomy. These are five dimensional black holes, which are characterized by a membrane charge $Q \in H_{2}(X, \mathbb{Z})$ and an angular momentum $m$. It was proposed in [28] that the microscopic entropy of these black holes is accounted for by BPS states associated to M2 branes wrapping the cycle $Q$ and with left spin $m=2 j_{L}$ in five dimensions. Accoording to the proposal of [28] their degeneracies are encoded in the five dimensional supersymmetric index

$$
I(\alpha, \beta)=\operatorname{Tr}(-1)^{2 j_{L}+2 j_{R}} \exp \left(-\alpha j_{L}-\beta H\right) .
$$


The information captured by this index can be extracted from the all-genus expansion of the holomorphic free energy of the topological string, computed at the large radius point of $X$ [21],

$$
\lim _{\bar{t} \rightarrow \infty} F\left(t, \bar{t}, g_{s}\right)=\sum_{g=0}^{\infty} g_{s}^{2 g-2} F_{g}(t) .
$$

In this identification the BPS degeneracies are mapped to the Gromov-Witten invariants of genus $g$ holomorphic maps. Since the topological string could not be solved on compact Calabi-Yau threefolds at higher genus, progress in understanding the microscopic degeneracies in the general case was very limited. On the boundary of the Kähler cone the problem might reduce effectively to a counting problem on a two complex dimensional surface, which is mathematically simpler, but the situation is also physically more degenerate. When the compactification manifold is $X=X_{2} \times \mathbb{T}^{2}$, where $X_{2}=\mathbb{T}^{4}$ or $\mathrm{K} 3$, one obtains the five dimensional black hole solutions constructed in [5], and the microscopic degeneracies are encoded in the elliptic genus of symmetric products of $X_{2}$.

In this paper we study the microscopic counting proposed in [28] in two different situations, by using numerical and analytic methods. First of all, we consider $5 \mathrm{~d}$ black holes obtained by compactifying M-theory on the one-parameter Calabi-Yau spaces studied in [26]. This explores a generic direction in the Kähler cone and allows to describe generic $5 \mathrm{~d}$ black holes, which have non-vanishing classical horizon area and can carry spin. In [26] significant progress was made in solving the topological string on compact CalabiYau threefolds. By combining the holomorphic anomaly of [4] with modularity properties of the topological string partition function $Z=\exp (F)$, effective action arguments, and Castelnouvo theory, it was possible to calculate the topological string free energy up to genus 53.

In order to make contact with black hole physics on the (super)gravity side, one has to obtain the asymptotic expansion of the microscopic degeneracies for large charge $Q$ and $Q \gg m$. For fixed $g$ the expansion of $F_{g}(t)$ around large radius is convergent and under analytic control by mirror symmetry. In contrast, the genus expansion in (1.2) is expected to be asymptotic, as in noncritical string theories [38] (see [33] for a recent discussion of this issue in the context of topological strings). To obtain a large $Q$ expansion for the degeneracies of the $(Q, m)$ states one needs information at genus $g \sim Q^{2}$ and is hence facing the issues of the behavior of string theory at large genus. Although we don't have enough control of the degeneracies to obtain analytical results on the large charge expansion, the situation is suited to a numerical study by using the method of Richardson transforms. This method merely relies on the knowledge of the form of the series and makes it possible to extract its coefficients from the value of the degeneracies at finitely many points. The analysis is complicated by the fact that the large charge expansion of the degeneracies is an asymptotic expansion, but we find that the Richardson transforms converge rapidly to the expected macroscopic values

\footnotetext{
${ }^{1}$ For sub-sub leading terms we use the Padé approximation.
} 
for the asymptotic coefficients. To estimate its accuracy we sample over the thirteen Calabi-Yau, which have a sufficently wide variety of topological data. Using this sample we can conclude that, given our present data of the higher genus instanton expansion, the leading coefficient of the asymptotic expansion is correct within $2 \%$ and the first subleading one within $12 \%$. With this information at hand, we give convincing evidence that the topological string accounts correctly for the entropy of $5 \mathrm{~d}$ spinning black holes, as conjectured in $[28]^{2}$.

Some aspects of the genus expansion (1.2) are much better understood in terms of D-brane invariants like Gopakumar-Vafa or Donaldson-Thomas invariants, rather than Gromov-Witten invariants. In particular, for a given charge $Q \in H_{2}(X, \mathbb{Z})$ and $Q \neq 0$ one gets the complete genus information from a finite number of GV invariants. We use the results for $F_{g}$ in [26] to obtain precise information on the Donaldson-Thomas invariants of the one-parameter models. This allows us to study numerically the scaling exponent $k$ considered in [12] (and defined below in (4.7) and (4.8)), which governs the growth of the Donaldson-Thomas invariants under rescalings of the charges. Our numerical study indicates that $k=2$. As argued in [12], this value indicates that highly nontrivial cancellations occur between the contributions to the Donaldson-Thomas invariants, which in turn seem necessary to resolve the so called entropy enigma [12] in the OSV conjecture 37.

The second class of black holes we study has a different flavor. These are $5 \mathrm{~d}$ black holes which are obtained when the Calabi-Yau is a K3 fibration and the charge $Q$ is restricted to the K3 fiber. Their classical horizon area is zero (i.e. they are small black holes) and have no spin. By using heterotic/type II duality one can obtain analytic formulae for the $F_{g}$ amplitudes at all $g$ [2, 34, 30, 32, 22], and from them one can extract the exact microscopic degeneracies for the corresponding small $5 \mathrm{~d}$ black holes. Of course, as explained for example in [12, the most delicate aspects of $5 \mathrm{~d}$ spinning black holes, as well as of the OSV conjecture, cannot be tested with small black holes. This reflects the fact that the Gromov-Witten theory of K3 fibers (which is closely related to the theory of Hilbert schemes) is much simpler than the Gromov-Witten theory of generic CalabiYau manifolds. However, having an exact microscopic counting might be important in understanding some detailed aspects of the entropy. As in the 4d case considered in [9], the $5 \mathrm{~d}$ degeneracies are closely related to modular forms, but one cannot use the Rademacher expansion featured in [15, 9]. We find however an exact asymptotic expansion for the microscopic degeneracies in powers of the inverse charge (albeit corrected by terms which are exponentially suppressed for large charges). The leading term of the asymptotics is in agreement with a macroscopic computation using the $4 \mathrm{~d} / 5 \mathrm{~d}$ connection of [20] and the 4d attractor equations for a D6/D2 system.

The organization of this paper is as follows. In section 2 we review the macroscopic and microscopic computation of the entropy for $5 \mathrm{~d}$ spinning black holes. In section 3

\footnotetext{
${ }^{2}$ For a recent study of this question by using an approach totally different from ours, see [24].
} 
we analyze numerically the asymptotic properties of the degeneracies for the Calabi-Yau manifolds studied in [26]. In section 4 we study the asymptotic properties of DonaldsonThomas invariants to address the entropy enigma of [12]. In section 5 we study small black holes in K3 fibrations and compute their degeneracies as well as the asymptotic expansion. Finally, in section 6 we list some conclusions and open problems.

\section{Microscopic and macroscopic counting for 5d black holes}

\subsection{Macroscopic description}

Let us start with the macroscopic description of black hole entropy. We will consider 5 d black holes obtained by compactifying $\mathrm{M}$ theory on a Calabi-Yau threefold $X$, and characterized by a charge $Q \in H_{2}(X, \mathbb{Z})$ and $S U(2)_{L} \subset S O(4)$ angular momentum $m$. We will introduce a basis $\Sigma^{A}$ for $H_{2}(X, \mathbb{Z})$, where $A=1, \cdots, b_{2}(X)$, as well as a dual basis $\eta_{A}$ for $H^{2}(X)$. With respect to the $\Sigma^{A}$ basis, the charge $Q$ will be given by a set of integers $Q_{A}$. The classical entropy of these black holes, denoted as $S_{0}$, is one quarter of the horizon area

$$
S_{0}=2 \pi \sqrt{\mathcal{Q}^{3}-m^{2}}
$$

where $\mathcal{Q}$ is the graviphoton charge of the black hole. This charge is related to the membrane charge $Q$ by the attractor mechanism in five dimensions [19], which states that

$$
\mathcal{Q}^{3 / 2}=\frac{1}{6} C_{A B C} y^{A} y^{B} y^{C}
$$

where

$$
\frac{1}{2} C_{A B C} y^{B} y^{C}=Q_{A}
$$

and

$$
C_{A B C}=\int_{X} \eta_{A} \wedge \eta_{B} \wedge \eta_{C}
$$

are the triple intersection numbers of $X$. For one-parameter models, the membrane charge will be identified with the degree $d$ of the holomorphic map in topological string computations, and we will denote the single intersection number by $C_{A B C}=\kappa$. From the above equations it follows that

$$
\mathcal{Q}=\left(\frac{2}{9 \kappa}\right)^{\frac{1}{3}} d .
$$

There is a correction to the black hole entropy from the $R^{2}$ term of the supergravity effective action, which we denote as $S_{1}$ for convenience. The $R^{2}$ term correction to the 
black hole entropy scales like $\mathcal{Q}^{\frac{1}{2}}$ in the large charge limit, and was computed in [23] by using Wald's formula [41] for the $R^{2}$ in 5 d. The result reads,

$$
S_{1}=\frac{\pi}{24} \sqrt{Q^{3}-m^{2}} c_{2} \cdot Y\left(\frac{3}{Q}+\frac{m^{2}}{Q^{4}}\right)
$$

where

$$
Y^{A}=\frac{1}{\mathcal{Q}^{1 / 2}} y^{A}
$$

and

$$
c_{2 A}=\int_{X} c_{2}(X) \wedge \eta_{A}
$$

For $m=0$ this formula has been rederived in [8, 1] by using the full 5d SUGRA action. In the one-parameter case, this correction reads

$$
S_{1}=\frac{\pi c_{2}}{8}\left(\frac{6}{\kappa}\right)^{\frac{1}{3}} \sqrt{Q^{3}-m^{2}}\left(\frac{1}{Q}+\frac{m^{2}}{3 Q^{4}}\right)
$$

Besides the corrections that we have considered, there are well known correction terms in the low energy effective action of the form $F^{2 g-2} R^{2}, g \geq 2$, where $F$ is the graviphoton field strength. The leading contribution comes from a classical term, which is the contribution of the constant map from a genus $g$ world-sheet to the Calabi-Yau manifold. It is of the form,

$$
d_{g} \chi
$$

where $\chi$ is the Euler number of the Calabi-Yau 3-fold and

$$
d_{g}=\frac{(-1)^{g}\left|B_{2 g} B_{2 g-2}\right|}{4 g(2 g-2)(2 g-2) !} .
$$

We denote the correction to black hole entropy due to the $F^{2 g-2} R^{2}$ term as $S_{g}$. We can roughly estimate the correction for non-spinning black holes $m=0$ as follows.

The graviphoton charge is the integral of its field strength over the horizon of black hole,

$$
\mathcal{Q} \sim \int_{\text {Horizon }} F
$$

Since the area of the horizon scales like $A \sim \mathcal{Q}^{\frac{3}{2}}$, the graviphoton field strength goes like

$$
F \sim \mathcal{Q}^{-\frac{1}{2}}
$$

The $R^{2}$ term contributes a factor $\mathcal{Q}^{-1}$ in Wald's formula for the black hole entropy, and taking into account also the factor of horizon area $A \sim \mathcal{Q}^{\frac{3}{2}}$, we find the scaling behavior of the $F^{2 g-2} R^{2}$ term correction to black hole entropy to be

$$
S_{g} \sim \chi \mathcal{Q}^{\frac{3}{2}-g}
$$


where we have included the Euler number from (2.10). The constant of proportionality in (2.14) is now universal, and independent of specific Calabi-Yau geometries and the black hole charge. We will be able to make a rough test of (2.14) for the genus 2 case, which is the sub-sub-leading correction in the large $\mathcal{Q}$ limit.

There are other worldsheet instanton corrections to the low energy effective action that can be computed also by topological strings. However, these terms are exponentially small in large charge $\mathcal{Q}$ limit where the supergravity description is valid, and are much suppressed compared to the $\mathcal{Q}^{-1}$ power corrections in (2.14). In this paper we will not need to consider these world-sheet instanton corrections in the macroscopic description of the black hole entropy. Interestingly these world-sheet instanton corrections are closely related to the BPS states that we will count in the microscopic description of the black hole entropy.

\subsection{Microscopic description}

Microscopically, a $5 \mathrm{~d}$ black hole with membrane charge $Q \in H_{2}(X, \mathbb{Z})$ is engineered by wrapping M2 branes around the two-cycle $Q$. This leads to a supersymmetric spectrum of BPS states in $5 \mathrm{~d}$ which are labeled by $Q$ and by their spin content $\left(j_{L}, j_{R}\right)$. As argued in [21], in order to obtain an index one has to trace over $j_{R}$ (with an insertion of $(-1)^{2 j_{R}}$ ). The resulting spectrum for a membrane charge $Q$ can be represented as

$$
R_{Q}=\sum_{r=0}^{g} n_{Q}^{r} I_{r+1}
$$

where

$$
I_{\ell}=\left[2(\mathbf{0})+\left(\frac{\mathbf{1}}{\mathbf{2}}\right)\right]^{\ell}
$$

encodes the spin content $j_{L}$, and $n_{Q}^{r}$ are the Gopakumar-Vafa invariants [21]. Notice that in (2.15), the sum over $r$ is finite and the highest spin $g$ appearing in the sum depends on the membrane charge $Q$. In other words, for any given $Q$ there are only finitely many $g$ for which the $n_{Q}^{r}$ are nonzero.

We can now write down a generating function for the supersymmetric degeneracies of BPS states with membrane charge $Q$, keeping track of their left spin $j_{L}$, by computing

$$
\sum_{m} \Omega(Q, m)=\sum_{Q} \operatorname{tr}_{R_{Q}}(-1)^{2 j_{L}} y^{j_{L}}
$$

Using the decomposition (2.15) one finds

$$
\Omega(Q, m)=\sum_{r}\left(\begin{array}{c}
2 r+2 \\
m+r+1
\end{array}\right) n_{Q}^{r} .
$$


where $m=2 j_{L}$. In [28] it was proposed that this quantity gives the microscopic degeneracies for a spinning $5 \mathrm{~d}$ black hole of charge $Q$ and spin $J=m$. The computation of these degeneracies reduces then to the computation of the Gopakumar-Vafa invariants $n_{Q}^{r}$. The most effective way to determine these is by computing the genus $r$ amplitudes $F_{r}$ of topological string theory on $X$. As shown in [21], the total free energy

$$
F\left(t, g_{s}\right)=\sum_{r=0}^{\infty} F_{r}(t) g_{s}^{2 r-2}
$$

can be written in terms of the Gopakumar-Vafa invariants as

$$
F\left(t, g_{s}\right)=\sum_{r=0}^{\infty} \sum_{Q \in H_{2}(X)} \sum_{k=1}^{\infty} n_{Q}^{r} \frac{1}{k}\left(2 \sin \frac{k g_{s}}{2}\right)^{2 r-2} \mathrm{e}^{-k Q \cdot t} .
$$

This means, in particular, that one can obtain the $n_{Q}^{r}$ for all $Q$ by knowing $F_{0}, \cdots, F_{r}$. The black hole entropy is given by logarithm of the number of microstates

$$
S(Q, m)=\log (\Omega(Q, m)) .
$$

This should agree with the macroscopic result in the large charge limit $Q \gg 1$ and $Q \gg m$.

As explained in [28], this proposal for the microscopic counting of states of $5 \mathrm{~d}$ black holes can be regarded as a generalization of the elliptic genus, which computes BPS degeneracies of the D1-D5 system. Indeed, if one considers $M$ theory compactified on $X=\mathrm{K} 3 \times \mathbb{T}^{2}$, the generic M2 brane charge in this compactification is

$$
Q=[C]+n\left[\mathbb{T}^{2}\right], \quad n \in \mathbb{Z},
$$

and $C$ is a curve in K3. By standard dualities this system can be related to type IIB on $\mathrm{K} 3 \times \mathbb{S}^{1}$ with $\mathrm{D}$-brane charge $[C]$ and $M$ units of momentum around $\mathbb{S}^{1}$, which is a close cousin of the D1-D5 system. As it is well known (see for example [14]), the BPS degeneracies of this system can be computed from the elliptic genus of the symmetric product of K3. Let

$$
\chi(\mathrm{K} 3 ; q, y)=\sum_{m, \ell} c(m, \ell) q^{m} y^{\ell}
$$

be the elliptic genus of K3. The generating function of elliptic genera of the symmetric product $S^{k} \mathrm{~K} 3$

$$
\chi\left(S_{p} \mathrm{~K} 3 ; q, y\right)=\sum_{k=0}^{p} \chi\left(S^{k} \mathrm{~K} 3 ; q, y\right) p^{k}=\sum_{k, n, m} c(k, n, m) p^{k} q^{n} y^{m}
$$

can be computed from the coefficients in (2.23) in terms of an infinite product [16]

$$
\chi\left(S_{p} \mathrm{~K} 3 ; q, y\right)=\prod_{N, M \geq 0, \ell}\left(1-p^{N} q^{M} y^{\ell}\right)^{-c(N M, \ell)} .
$$




\begin{tabular}{|r|rrr||r|rrr|}
\hline CY & $\chi$ & $c_{2} \cdot \eta$ & $\kappa$ & CY & $\chi$ & $c_{2} \cdot \omega$ & $\kappa$ \\
\hline$X_{5}\left(1^{5}\right)$ & -200 & 50 & 5 & $X_{6}\left(1^{4}, 2\right)$ & -204 & 42 & 3 \\
$X_{8}\left(1^{4}, 4\right)$ & -296 & 44 & 2 & $X_{10}\left(1^{3}, 2,5\right)$ & -288 & 34 & 1 \\
$X_{3,3}\left(1^{6}\right)$ & -144 & 54 & 9 & $X_{4,2}\left(1^{6}\right)$ & -176 & 56 & 8 \\
$X_{3,2,2}\left(1^{7}\right)$ & -144 & 60 & 12 & $X_{2,2,2,2}\left(1^{8}\right)$ & -128 & 64 & 16 \\
$X_{4,3}\left(1^{5}, 2\right)$ & -156 & 48 & 6 & $X_{4,4}\left(1^{4}, 2^{2}\right)$ & -144 & 40 & 4 \\
$X_{6,2}\left(1^{5}, 3\right)$ & -256 & 52 & 4 & $X_{6,4}\left(1^{3}, 2^{2}, 3\right)$ & -156 & 32 & 2 \\
$X_{6,6}\left(1^{2}, 2^{2}, 3^{2}\right)$ & -120 & 32 & 1 & & & & \\
\hline
\end{tabular}

Table 3.1: The sample of 13 one-parameter complete intersection CYs in weighted projective spaces. A CICY of degree $d_{1}, \ldots, d_{k}$ in weighted projective space $\mathbb{P}^{l-1}\left(w_{1}, \ldots, w_{l}\right)$ is denoted $X_{d_{1}, \ldots, d_{k}}\left(w_{1}, \ldots, w_{l}\right)$, i.e. weights $w$ with repetition $m$ are abbreviated by $w^{m}$. $\chi=\int_{X} c_{3}$ is the Euler number, $\kappa$ is the triple intersection number, and $c_{2} \cdot \eta$ is defined in $(2.8)$.

The supersymmetric degeneracies of BPS states for this system are then given by the coefficients of the expansion in (2.24),

$$
\Omega(Q, m)=c\left(\frac{1}{2} C^{2}+1, n, m\right),
$$

where $Q$ is of the form (2.22). One can show that, for large charges [17, 14],

$$
\log \Omega(Q, m) \sim 2 \pi \sqrt{\frac{n}{2} C^{2}-m^{2}} .
$$

It is easy to check that this is precisely the macroscopic entropy (2.1) computed for $\mathrm{K} 3 \times \mathbb{T}^{2}$. Of course, the degeneracies (2.18) are in general much more difficult to compute, since they correspond to black holes with only $\mathcal{N}=1$ supersymmetry in $5 \mathrm{~d}$.

\section{One-parameter models}

\subsection{Topological strings on one--parameter models}

In 26] the topological B model was integrated on thirteen one-parameter Calabi-Yau spaces which can be realized as hypersurfaces or complete intersections in (weighted) projective spaces. We have listed these spaces and some of their topological data in table 3.1. These data are the intersection numbers $C_{A B C}=\kappa$, the second Chern classes $c_{2}$, and the Euler numbers $\chi$. They are needed for computations of the macroscopic entropy.

The complex moduli space of these threefolds is $\mathcal{M}=\mathbb{P}^{1} \backslash\{\infty, 1,0\}$, and the three special points are the large radius degeneration point, a conifold point and a further point either of finite (Gepner point) or infinite branching order. The modular group $\Gamma_{X} \in$ $\mathrm{SP}(4, \mathbb{Z})$ can hence be generated e.g. by the large radius and the conifold monodromies. 
The conceptual obstacle in integrating the B-model holomorphic anomaly [4] is the holomorphic ambiguity which arises in each integration step. Invariance of the topological string amplitudes under $\Gamma_{X}$ and effective action arguments, which govern the behaviour of the genus $g$ amplitudes at special points, restrict the ambiguity to $3 g-3$ unknowns [28]. By using a refined effective action analysis, which gives rise to the "gap condition" at the conifold, regularity at the orbifold, and Castelnouvo's bound for the Gromow-Witten invariants at large radius, it is possible to fix the unknows, and one can calculate the free energy of the topological string to arbitrary degree and up to genus $12-53$.

Instead of using the generic solution of holomorphic anomaly equation suggested by the world-sheet degenerations [4] we use the constraints of $\Gamma_{X}$ on the topological string amplitudes directly when integrating the holomorphic anomaly equations genus by genus [42] 22]. This results in an alogarithm, which constructs the genus $g$ amplitudes as weight $3 g-3$ polynomials over a ring of three an-holomorphic- and one holomorphic modular objects of weight $(1,2,3,1)$. As a consequence the number of terms in the $F_{g}$ grows polynomial with $g$ and not exponentially as in the approach of $[4]^{3}$.

The approach of [26] views the topological string partition function as a wave function over $H^{3}(X, \mathbb{R})$. Choices of polarization are necessary in order to expand the effective action at different points in the moduli space $\mathcal{M}$, in appropriate local holomorphic coordinates. Most of the black hole issues that we will discuss involve the degeneracies extracted from the topological string at the large radius limit. Therefore we will discard for now most of the global information and focus only the holomorphic limit of the topological string partition function at this limit, where it encodes the degeneracy of bound states of a single D6 brane and arbitrary D2-D0 branes.

\subsection{Static black holes}

We first consider the case of non-spinning black hole $J \equiv m=0$ and denote $N_{d}=\Omega(d, 0)$. The entropy formula including the first few orders (2.1), (2.9), (2.14) is in this case

$$
S=b_{0} d^{\frac{3}{2}}+b_{1} d^{\frac{1}{2}}+\frac{b_{2}}{d^{\frac{1}{2}}}+\mathcal{O}\left(\frac{1}{d^{\frac{3}{2}}}\right)
$$

where the first two coefficients are

$$
b_{0}=\frac{4 \pi}{3 \sqrt{2 \kappa}}, \quad b_{1}=\frac{\pi c_{2}}{4 \sqrt{2 \kappa}}
$$

and we have used the graviphoton charge relation (2.5). The coefficient $b_{1}$ of the subleading term is consistent with the results in [40, 8, 1, where it was observed that the $b_{1}$ can be produced by a shift of the 2-brane charge

$$
d \rightarrow d+\frac{c_{2}}{8}
$$

\footnotetext{
${ }^{3}$ Nevertheless the limiting factor in advancing to higher $g$ is presently not the ambiguity but the computing time. The reason is that the numerators in the coefficents of the polynomials grow exponentially.
} 
in the leading term.

To compare with the microscopic counting we define the following function

$$
f(d)=\frac{\log \left(N_{d}\right)}{d^{\frac{3}{2}}}
$$

The macroscopic black hole entropy predicts that the large order behavior of $f(d)$ is

$$
f(d)=b_{0}+\frac{b_{1}}{d}+\frac{b_{2}}{d^{2}}+\cdots
$$

Since we have available only the values of $f(d)$ for positive integer $d$ up to a finite degree, it is appropriate to use well-known numerical methods to extrapolate the asymptotic value $b_{0}$. From the form of the sub-leading corrections in (3.5), it is appropriate to use the Richardson extrapolation method (see for example [3]).

The basic idea of this numerical method is simple. To cancel the sub-leading corrections in (3.5) up to order $1 / d^{N}$, one defines

$$
A(d, N)=\sum_{k=0}^{N} \frac{f(d+k)(d+k)^{N}(-1)^{k+N}}{k !(N-k) !}
$$

One can show that if the perturbation series (3.5) truncates at order $1 / d^{N}$, the expression (3.6) will give exactly the asymptotic value $b_{0}$. Ideally , the larger $N$ and $d$ are, the closer $A(d, N)$ is to the asymptotic value. But due to the limitation of our data, the sum $d+N$ must not exceed the maximal degree $d_{\max }$ of the topological string computations.

Fig. 1 shows the convergence of the leading terms in $f(d)$ and of the Richardson transforms $A(d, N), N=2,3,4$ for the quintic and the bi-cubic. It is obvious from the two examples in Fig. 1 that the Richardson method improves impressively the convergence of the series, i.e. it provides a model independent and consistent scheme to supress the subleading corrections. Using $N=2-4$ is good enough for our purpose of estimating the asymptotic value. We conduct the analysis for all 13 models using $N=3$ and the maximal degree available. The results are summarized in Table 3.2 , and are in very good agreements with the expected asymptotic values $b_{0}$ in (3.2). More detailed results on all the analysis carried out in this paper can be found in a script and in a data base at [43].

We can further extract the sub-leading coefficient $b_{1}$ from the data. Define

$$
\begin{aligned}
f_{1}(d) & =\left(f(d)-b_{0}\right) d \\
A_{1}(d, N) & =\sum_{k=0}^{N} \frac{f_{1}(d+k)(d+k)^{N}(-1)^{k+N}}{k !(N-k) !}
\end{aligned}
$$

and the asymptotic value of $f_{1}(d)$ should be $b_{1}$. We apply the same Richardson extrapolation method to $f_{1}(d)$ and we compare it with the macroscopic black hole predictions. 

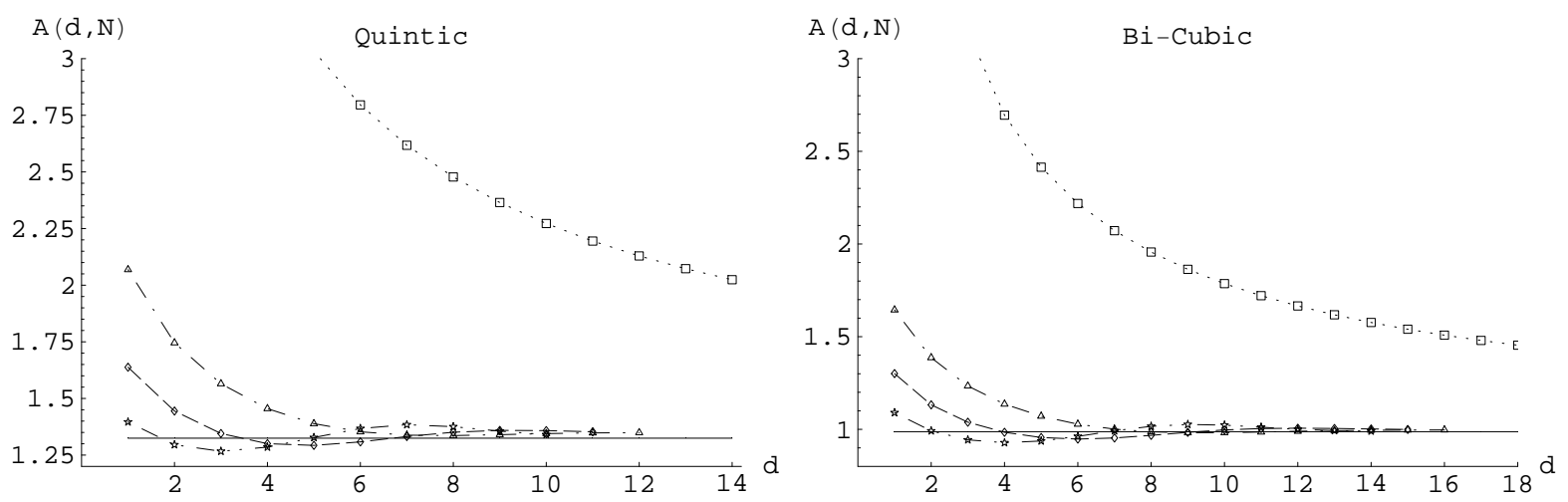

Figure 1: Microscopic data for $f(d)(\square)$, and the Richardson transforms $A(d, 2)(\triangle)$, $A(d, 3)(\diamond)$, and $A(d, 4)(\star)$. The straight line corresponds to the macroscopic prediction $b_{0}=\frac{4 \pi}{3 \sqrt{2 \kappa}}$. For the quintic this value is $b_{0} \approx 1.359$ and for the available degree 14 the Richardson transforms lie 1.8, 2.1, $1.2 \%$ from the macroscopic prediction. For the bicubic $b_{0} \approx 0.967$, the available degree is higher, 18 , and the microscopic counting is within $.9,1.2, .3 \%$ from the macroscopic prediction. As an example we give BPS numbers used for the analysis at degree 18 of the bi-cubic in table (A.1).

\begin{tabular}{|r|r|r|r|r|}
\hline Calabi-Yau & $d_{\max }$ & $A\left(d_{\max }-3,3\right)$ & $b_{0}=\frac{4 \pi}{3 \sqrt{2 \kappa}}$ & error \\
\hline$X_{5}\left(1^{5}\right)$ & 14 & 1.35306 & 1.32461 & $2.15 \%$ \\
\hline$X_{6}\left(1^{4}, 2\right)$ & 10 & 1.75559 & 1.71007 & $2.66 \%$ \\
\hline$X_{8}\left(1^{4}, 4\right)$ & 7 & 2.11454 & 2.0944 & $0.96 \%$ \\
\hline$X_{10}\left(1^{3}, 2,5\right)$ & 5 & 2.99211 & 2.96192 & $1.02 \%$ \\
\hline$X_{3,3}\left(1^{6}\right)$ & 17 & 1.00204 & 0.987307 & $1.49 \%$ \\
\hline$X_{4,2}\left(1^{6}\right)$ & 15 & 1.07031 & 1.0472 & $2.21 \%$ \\
\hline$X_{3,2,2}\left(1^{7}\right)$ & 10 & 0.821169 & 0.855033 & $-3.96 \%$ \\
\hline$X_{2,2,2,2}\left(1^{8}\right)$ & 13 & 0.722466 & 0.74048 & $-2.43 \%$ \\
\hline$X_{4,3}\left(1^{5}, 2\right)$ & 11 & 1.21626 & 1.2092 & $0.58 \%$ \\
\hline$X_{6,2}\left(1^{5}, 3\right)$ & 11 & 1.52785 & 1.48096 & $3.17 \%$ \\
\hline$X_{4,4}\left(1^{4}, 2^{2}\right)$ & 7 & 1.42401 & 1.48096 & $-3.85 \%$ \\
\hline$X_{6,4}\left(1^{3}, 2^{2}, 3\right)$ & 5 & 2.06899 & 2.0944 & $-1.21 \%$ \\
\hline$X_{6,6}\left(1^{2}, 2^{2}, 3^{2}\right)$ & 4 & 2.95082 & 2.96192 & $-0.37 \%$ \\
\hline
\end{tabular}

Table 3.2: Comparing the extrapolated value of $b_{0}$ with the macroscopic prediction. 

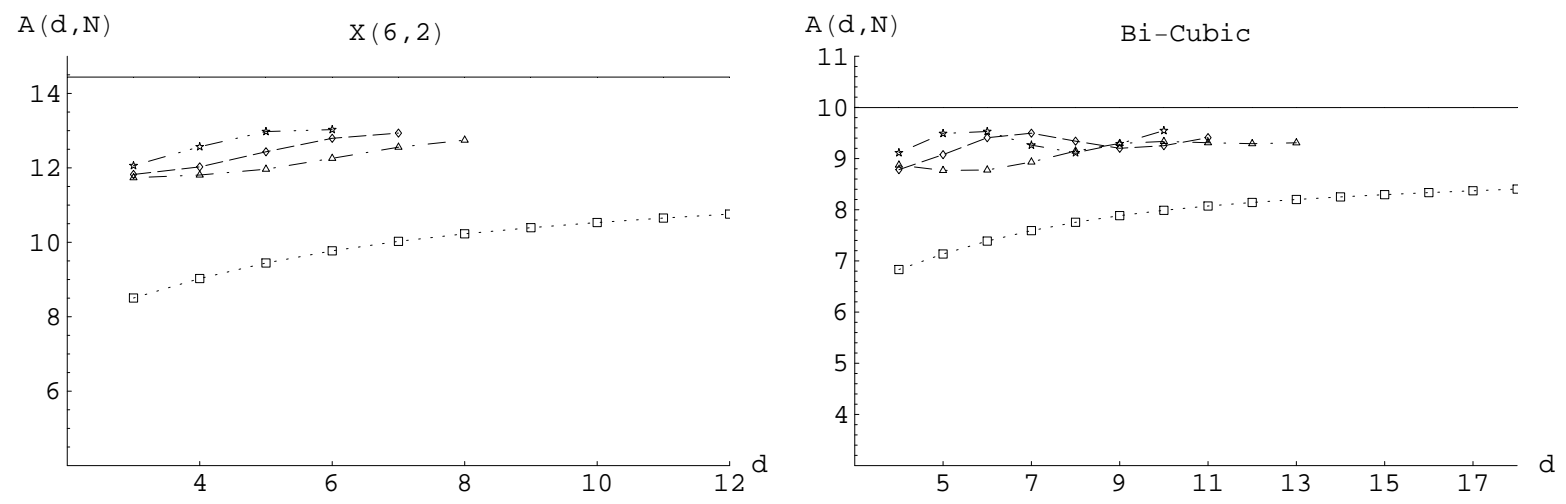

Figure 2: Microscopic data for $f(d)(\square)$, and the Richardson transforms $A(d, 4)(\triangle)$, $A(d, 5)(\diamond)$, and $A(d, 6)(\star)$. The straight line corresponds to the macroscopic prediction $b_{1}=\frac{\pi c_{2}}{4 \sqrt{2 \kappa}}$. For the degree $X_{6,2}$ complete intesection this value is $b_{1} \approx 14.44$ and for the available degree 12 the Richardson transforms lie $-11.7,-10.4,-9.77 \%$ below the macroscopic prediction. For the bi-cubic $b_{1} \approx 9.994$, the available degree is 18 and the microscopic counting is $-7.15,-6.88,-6.63 \%$ below the macroscopic prediction.

\begin{tabular}{|r|r|r|r|r|r|}
\hline Calabi-Yau & $d_{\max }$ & $A_{1}\left(d_{\max }-3,3\right)$ & $b_{1}=\frac{\pi c_{2}}{4 \sqrt{2 \kappa}}$ & error & estimated $b_{2}$ \\
\hline$X_{5}\left(1^{5}\right)$ & 14 & 11.2668 & 12.4182 & $-9.27 \%$ & -11.9503 \\
\hline$X_{6}\left(1^{4}, 2\right)$ & 10 & 11.9237 & 13.4668 & $-11.5 \%$ & -12.1848 \\
\hline$X_{8}\left(1^{4}, 4\right)$ & 7 & 14.0537 & 17.2788 & $-18.7 \%$ & -14.9973 \\
\hline$X_{10}\left(1^{3}, 2,5\right)$ & 5 & 15.2509 & 18.8823 & $-19.2 \%$ & -14.9817 \\
\hline$X_{3,3}\left(1^{6}\right)$ & 17 & 9.29062 & 9.99649 & $-7.06 \%$ & -9.63958 \\
\hline$X_{4,2}\left(1^{6}\right)$ & 15 & 10.0226 & 10.9956 & $-8.85 \%$ & -10.7834 \\
\hline$X_{3,2,2}\left(1^{7}\right)$ & 10 & 8.45163 & 9.61912 & $-12.1 \%$ & -9.3828 \\
\hline$X_{2,2,2,2}\left(1^{8}\right)$ & 13 & 7.84595 & 8.88577 & $-11.7 \%$ & -8.88773 \\
\hline$X_{4,3}\left(1^{5}, 2\right)$ & 11 & 9.5981 & 10.8828 & $-11.8 \%$ & -9.96404 \\
\hline$X_{6,2}\left(1^{5}, 3\right)$ & 11 & 12.5614 & 14.4394 & $-13.0 \%$ & -14.2582 \\
\hline$X_{4,4}\left(1^{4}, 2^{2}\right)$ & 7 & 9.70091 & 11.1072 & $-12.7 \%$ & -9.41295 \\
\hline$X_{6,4}\left(1^{3}, 2^{2}, 3\right)$ & 5 & 11.1008 & 12.5664 & $-11.7 \%$ & -10.0821 \\
\hline$X_{6,6}\left(1^{1}, 2^{2}, 3^{3}\right)$ & 4 & 11.1378 & 12.2179 & $-8.84 \%$ & -8.15739 \\
\hline
\end{tabular}

Table 3.3: Comparing the extrapolated value with the macroscopic prediction of $b_{1}$. 
Two typical examples for the behaviour of the Richardson transforms are plotted in Fig. 2. The results for all models are summarized in Table 3.3 .

Despite our rather successful verifications of the numerical coefficients $b_{0}$ and $b_{1}$, we should note that the expansion in inverse powers of the charge (3.5) is actually an asymptotic series. The asymptotic character of the large charge expansions of microscopic degeneracies is manifest in the explicit computations for small black holes in [9] and also in the examples we will discuss in section 5 . In our case, we can relate the asymptotic expansion of (3.5) to a large genus behavior in a string series, since the coefficients in (3.5) are proportional to the constant map contribution

$$
b_{g} \sim d_{g}
$$

where $d_{g}$ is given in (2.11). This coefficient grows at large $g$ as

$$
d_{g} \sim(2 \pi)^{-4 g}(-1)^{g}(2 g) !
$$

which is the typical behavior found in string perturbation theory [38]. It then follows that the series expansion (3.5) for $f(d)$ has zero radius of convergence for any value of $d$ and it is rather an asymptotic expansion. Indeed, the $d_{g}$ are the coefficients of the asymptotic expansion of the MacMahon function (see [9], Appendix E, for a detailed derivation). For these kinds of expansions, the best approximation to their true value (which in this case is the function $f(d)$ computed from topological strings) is obtained by truncating the sum at the order $\mathcal{N}$ which minimizes the error. For an asymptotic series of the form

$$
f(w)=\sum_{k=1}^{\infty} b_{k} w^{k}, \quad b_{k} \sim A^{-k}(\beta k) !
$$

the optimal truncation occurs generically at

$$
\mathcal{N} \sim \frac{1}{\beta}\left(\frac{A}{|w|}\right)^{\frac{1}{\beta}} .
$$

In our case $\beta=2$ and we can estimate $\mathcal{N}$ as follows. According to the connection between $4 \mathrm{D} / 5 \mathrm{D}$ black holes [20], the attractor value for the topological string coupling constant is $g_{s}=4 \pi$ [23]. This should be roughly the numerical constant that relates the graviphoton field strength to the charge $\mathcal{Q}$ in (2.13), and it contributes to the coefficients $b_{g}$ an extra factor $g_{s}^{2 g-2}$, so that we can refine (3.8) to

$$
b_{g} \sim(4 \pi)^{2 g} d_{g}
$$

and the constant in (3.10) is $A=\pi^{2}$. Therefore, the optimal truncation is at

$$
\mathcal{N} \sim \frac{\pi}{2} d^{\frac{1}{2}}
$$


For the small values of $d$ that we are considering we should therefore expect an optimal truncation around $\mathcal{N} \sim 5-10$.

These considerations have implications for our numerical analysis. The Richardson method (3.6) is designed in principle for convergent expansions. For asymptotic expansions, we should expect it to give increasing precision and convergence to the true coefficients as long as the order of the transformation $N$ in (3.6) is lower than the truncation order $\mathcal{N}$. This is the underlying reason that prevents us from improving the precision of the leading coefficients by simply increasing the truncation order $N$ in the Richardson method, and we indeed find an oscillating behavior around the expected true value for the Richardson transforms with $N>5$.

We try to go one step further and give a rough estimation of the coefficient $b_{2}$ in (3.1), which has not been studied in the literature from the supergravity point of view. It turns out that the naive method we use for computing the sub-leading coefficient $b_{1}$ gives too big an estimate, which might be a result that the optimal truncation scheme is no longer a good approximation at this order. In order to improve this, we use the Padé approximation which is well-known for summing divergent series. Given an asymptotic series

$$
f(z)=\sum_{i=0}^{\infty} b_{i} z^{i},
$$

one can evaluate the asymptotic value by defining the following Padé approximation

$$
P_{M}^{N}(z)=\frac{\sum_{i=0}^{N} A_{i} z^{i}}{1+\sum_{i=1}^{M} B_{i} z^{i}}
$$

where the coefficients $A_{i}$ and $B_{i}$ are fixed by Taylor expanding the above equation (3.15) around $z=0$ and match to the first $M+N+1$ terms of the original series (3.14).

We take the theoretical values of $b_{0}$ and $b_{1}$ from (3.2), and use the Monte Carlo method to randomly generate the sub-leading coefficients $b_{2}, b_{3}$ etc, then use the Padé approximation to evaluate the asymptotic series (3.14) for $z=\frac{1}{d}$, where $d=1,2, \cdots, d_{\max }$. We pick the sub-leading coefficients $b_{i}(i \geq 2)$ that minimize the difference of the Padé evaluation with the expected value $f(d)$ from topological strings, i.e. we minimize

$$
\sum_{d=1}^{d_{\max }}\left(\frac{P_{M}^{N}\left(\frac{1}{d}\right)}{f(d)}-1\right)^{2}
$$

We find different values of $N, M$ in the Padé approximation give qualitatively similar results. In the last column in Table 3.3, we give the estimated values of sub-sub-leading coefficient $b_{2}$ using the scheme $N=2, M=1$.

Assuming the constant map contribution is the most significant contribution at this order in $\mathcal{Q}$, the coefficient $b_{2}$ should behave like

$$
b_{2} \sim \chi \kappa^{\frac{1}{6}} .
$$




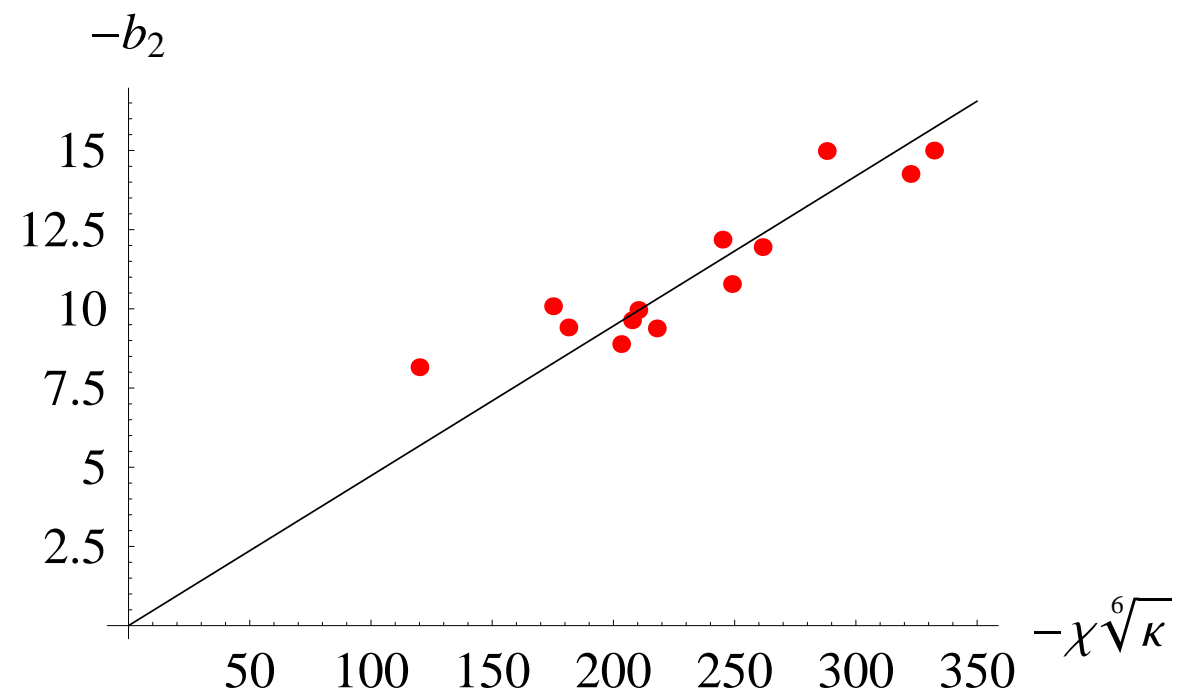

Figure 3: The plot of $-b_{2}$ vs. $\left(-\chi \kappa^{\frac{1}{6}}\right)$ for 13 Calabi-Yau models.

We can verify the relation (3.17) by plotting $b_{2}$ against the Euler number $\chi^{\frac{1}{6}}$ for the 13 Calabi-Yau models we studied. We find as the best fit coefficient

$$
b_{2}=0.047 \chi \kappa^{\frac{1}{6}}
$$

see the plot in Figure 3, which is reasonably consistent with the expectation (3.17).

From the second row of the Table 3.8, we can find the numerical values of the genus two constant map contribution $b_{2} \sim 0.00017 g_{s}^{2} \chi \kappa^{\frac{1}{6}}$. Taking into account that $g_{s} \sim \mathcal{O}(10)$, this is the same order of magnitude as our estimate value of 0.047 from microscopic topological string computation.

\subsection{Spinning black holes}

We can try to extract the spin dependence of the black hole entropy from (2.1). Assuming $\mathcal{Q} \gg J$, and expanding in $J / \mathcal{Q}$, we find the following macroscopic prediction for the topological string data,

$$
g_{m}(d) \equiv \frac{d^{\frac{3}{2}}}{m^{2}} \log \left(\frac{\Omega(d, 0)}{\Omega(d, m)}\right)=p_{0}+\mathcal{O}\left(\frac{1}{d}\right)
$$

where

$$
p_{0}=3 \pi\left(\frac{\kappa}{2}\right)^{\frac{1}{2}}
$$




\begin{tabular}{|r|r|r|r|r|r|r|}
\hline$d$ & $g_{1}(d)$ & $A_{1}(d, 1)$ & $g_{2}(d)$ & $A_{2}(d, 1)$ & $g_{3}(d)$ & $A_{3}(d, 1)$ \\
\hline 1 & 0.693147 & 3.22789 & $\mathrm{NA}$ & $\mathrm{NA}$ & $\mathrm{NA}$ & $\mathrm{NA}$ \\
\hline 2 & 1.96052 & 6.85432 & $\mathrm{NA}$ & $\mathrm{NA}$ & $\mathrm{NA}$ & $\mathrm{NA}$ \\
\hline 3 & 3.59178 & 10.9389 & 9.03347 & 12.2117 & $\mathrm{NA}$ & $\mathrm{NA}$ \\
\hline 4 & 5.42856 & 14.4696 & 9.82804 & 13.0403 & 12.1257 & 6.55334 \\
\hline 5 & 7.23677 & 16.4156 & 10.4705 & 12.8183 & 11.0112 & 10.2148 \\
\hline 6 & 8.76658 & 16.1819 & 10.8618 & 11.6135 & 10.8785 & 9.98996 \\
\hline 7 & 9.82591 & 13.9173 & 10.9692 & 9.71239 & 10.7516 & 8.81357 \\
\hline 8 & 10.3373 & 10.4832 & 10.8121 & 7.53259 & 10.5093 & 7.27017 \\
\hline 9 & 10.3535 & 7.02869 & 10.4477 & 5.51774 & 10.1494 & 5.73628 \\
\hline 10 & 10.021 & 4.41912 & 9.9547 & 3.9872 & 9.70809 & 4.46946 \\
\hline 11 & 9.51178 & 2.9195 & 9.4122 & 3.04128 & 9.23185 & 3.58335 \\
\hline 12 & 8.96242 & $\mathrm{NA}$ & 8.88129 & $\mathrm{NA}$ & 8.76114 & $\mathrm{NA}$ \\
\hline
\end{tabular}

Table 3.4: The Richardson method for the quintic with spin $m=1,2,3$.

For a fixed value $m$ we use again the Richardson extrapolation method to find the asymptotic value of $g_{m}(d)$ for large $d$. We list the values of $g_{m}(d)$ and its first Richardson extrapolation $A_{m}(d, 1)$ for spin $m=1,2,3$, using the quintic as an example.

We note that the contribution to entropy from angular momentum is proportional to $d^{-3 / 2}$, as compared to the leading static contribution (3.1) of order $d^{3 / 2}$. Although the prediction (3.19) should be the leading spinning contribution, there could be some small statistical fluctuation of topological string data which is random for the different spins, and which might become comparable to the spinning contribution in (3.19) and result in the deviation for large degree $d$. This can be seen in the quintic example in Table 3.4. We find that the Richardson series does not converge to an asymptotic value, instead the series approach a maximal value before deviating again for large degree $d$. In order to minimize the effect of statistical fluctuation of topological string data, we propose to use the extremal values in the Richardson series $A_{m}(d, 1)$ to estimate the asymptotic value of $p_{0}$. This is indeed a relatively good estimate for the quintic case where $p_{0}=14.9019$. Other Calabi-Yau manifolds are analyzed in [43].

We analyze the 13 Calabi-Yau models using the above approach. Let us define the extremal value of the first Richardson extrapolation over the degree $d$ as

$$
\tilde{g}_{m}=\left.A_{m}(d, 1)\right|_{\max },
$$

For various Calabi-Yau models and spin $m=1,2,3$, we compare the value of $\tilde{g}_{m}$ with the expected coefficient $p_{0}$ given in (3.20). The results are summarized in Table 3.5. We see that for larger angular momentum $m$ the deviations become bigger, as expected. 


\begin{tabular}{|r|r|r|r|r|r|r|r|}
\hline Calabi-Yau & $p_{0}=3 \pi\left(\frac{\kappa}{2}\right)^{\frac{1}{2}}$ & $\tilde{g}_{1}$ & $\tilde{g}_{1}$ error & $\tilde{g}_{2}$ & $\tilde{g}_{2}$ error & $\tilde{g}_{3}$ & $\tilde{g}_{3}$ error \\
\hline$X_{5}(1)$ & 14.9019 & 16.4156 & $10.2 \%$ & 13.0403 & $-12.5 \%$ & 10.2148 & $-31.5 \%$ \\
\hline$X_{6}\left(1^{4}, 2\right)$ & 11.5429 & 12.1492 & $5.25 \%$ & 10.1828 & $-11.8 \%$ & 8.21085 & $-28.9 \%$ \\
\hline$X_{8}\left(1^{4}, 4\right)$ & 9.42478 & 10.4854 & $11.3 \%$ & 8.1382 & $-13.7 \%$ & 5.3473 & $-43.3 \%$ \\
\hline$X_{10}\left(1^{3}, 2,5\right)$ & 6.66432 & 6.77436 & $1.65 \%$ & 5.89201 & $-11.6 \%$ & 3.62439 & $-45.6 \%$ \\
\hline$X_{3,3}\left(1^{6}\right)$ & 19.993 & 22.1786 & $10.9 \%$ & 17.7804 & $-11.1 \%$ & 14.8114 & $-25.9 \%$ \\
\hline$X_{4,2}\left(1^{6}\right)$ & 18.8496 & 21.0741 & $11.8 \%$ & 16.569 & $-12.1 \%$ & 12.9935 & $-31.1 \%$ \\
\hline$X_{3,2,2}\left(1^{7}\right)$ & 23.0859 & 25.9065 & $12.2 \%$ & 20.4996 & $-11.2 \%$ & 16.5636 & $-28.3 \%$ \\
\hline$X_{2,2,2,2}\left(1^{8}\right)$ & 26.6573 & 30.1999 & $13.3 \%$ & 23.6923 & $-11.1 \%$ & 19.2311 & $-27.9 \%$ \\
\hline$X_{4,3}\left(1^{5}, 2\right)$ & 16.3242 & 17.7685 & $8.85 \%$ & 14.4772 & $-11.3 \%$ & 12.2514 & $-24.9 \%$ \\
\hline$X_{6,2}\left(1^{5}, 3\right)$ & 13.3286 & 15.2332 & $14.3 \%$ & 11.2819 & $-15.4 \%$ & 8.06844 & $-39.5 \%$ \\
\hline$X_{4,4}\left(1^{4}, 2^{2}\right)$ & 13.3286 & 13.9081 & $4.35 \%$ & 11.618 & $-12.8 \%$ & 10.6901 & $-19.8 \%$ \\
\hline$X_{6,4}\left(1^{3}, 2^{2}, 3\right)$ & 9.42478 & 9.02611 & $-4.23 \%$ & 7.87731 & $-16.4 \%$ & 7.56862 & $-19.7 \%$ \\
\hline$X_{6,6}\left(1^{2}, 2^{2}, 3^{2}\right)$ & 6.66432 & 5.42333 & $-18.6 \%$ & 4.91355 & $-26.3 \%$ & 4.5984 & $-31.0 \%$ \\
\hline
\end{tabular}

Table 3.5: The Richardson method for the 13 Calabi-Yau models with spin $m=1,2,3$.

\section{Asymptotics of the Donaldson-Thomas invariants}

As we already mentioned, the total free energy of the topological string (2.19) can be reorganized in terms of Gopakumar-Vafa invariants as in (2.20). A remarkable property of $(2.20)$ is that for a given class $Q \in H_{2}(X, \mathbb{Z})$, the expression is exact in the string coupling. This is because Castelnuovo's theorem for the ambient space yields $n_{d}^{g}=0$ for $d>\alpha \sqrt{g}$ for certain $\alpha$.

For example, for the quintic the maximal genus $g_{\max }$ such that $n_{Q}^{g_{\max }} \neq 0$ fulfills a bound

$$
g_{\max } \leq \frac{1}{10}\left(10+5 d+d^{2}\right)
$$

with a decreasing relative deviation in the large $d$ limit. The bound is saturated for curves of total degree $5 m$ which are complete intersections of degree $(1,5, m)$ in $\mathbb{P}^{4}$, which are smooth curves in the quintic. For $5>m>1$ we can describe the moduli space of the D2 brane as follows. The linear constraint has as a parameter space $\mathbb{P}^{4}$ and allows to eliminate one variable from the degree $m$ constraint, which has as many homogeneous parameters as monomials in four variables, i.e. as many as there are integer solutions to $\sum_{i=1}^{4} n_{i}=m$ namely $\left(\begin{array}{c}m+4-1 \\ m\end{array}\right)$. The moduli spaces of the curves are therefore fibrations of $\mathbb{P}^{\left(\begin{array}{c}m+4-1 \\ m\end{array}\right)-1}$ over $\mathbb{P}^{4}$. Using the results of [28] we get for the GV invariant

$$
n_{5 m}^{g_{\max }}=(-1)^{\left(\begin{array}{c}
m+4-1 \\
m
\end{array}\right)-1} \cdot 5 \cdot\left(\begin{array}{c}
m+4-1 \\
m
\end{array}\right) \text {. }
$$

If the bound (4.1) is not saturated for small $d$ the relative deviation can become somewhat larger as seen in the Figure 4 . 


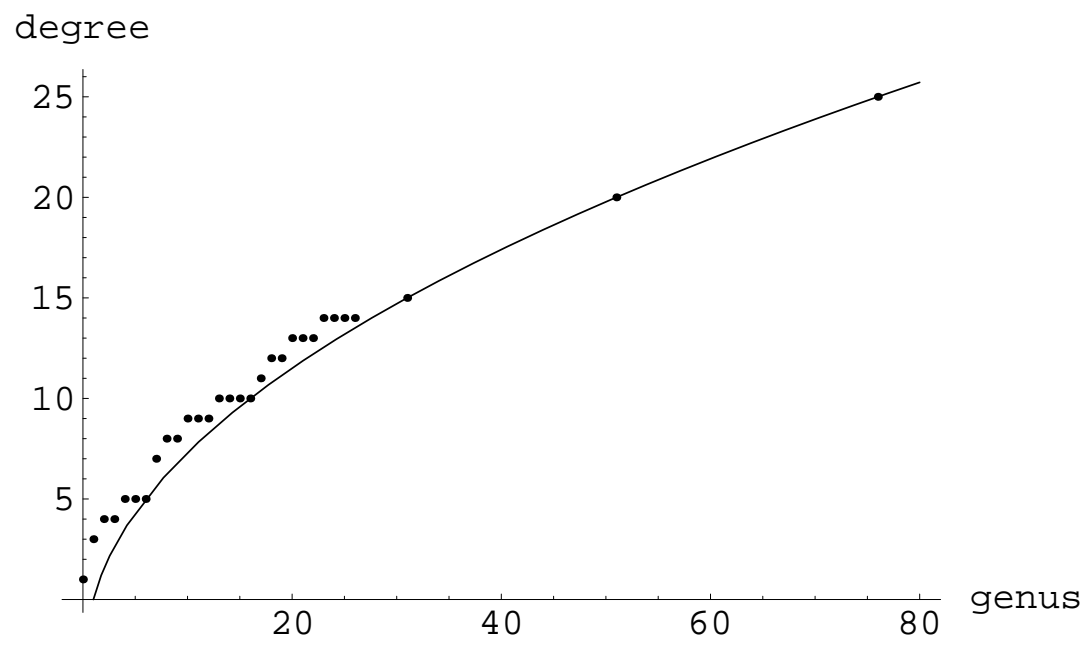

Figure 4: Castelnuovo's bound for higher genus curves on the quintic. The dots represent $n_{d}^{g_{\max }}$ and the curve is (4.1).

Let us denote by $F^{\prime}(\lambda, t)$ the total free energy without the contribution (2.10). After exponentiation one finds 30 ]

$$
Z_{\mathrm{GV}}^{\prime}(X, q, t)=\prod_{d=1}^{\infty}\left[\left(\prod_{r=1}^{\infty}\left(1-q^{r} \mathrm{e}^{-d t}\right)^{r n_{d}^{0}}\right) \prod_{g=1}^{\infty} \prod_{l=0}^{2 g-2}\left(1-q^{g-l-1} \mathrm{e}^{-d t}\right)^{(-1)^{g+l}\left(\begin{array}{c}
2 g-2 \\
l
\end{array}\right) n_{d}^{g}}\right],
$$

where

$$
q=\mathrm{e}^{\mathrm{i} \lambda}
$$

and we have assumed that there is only one Kähler parameter, so that $Q$ is labeled by a single integer $d$. On the other hand, the conjecture of [35] relating the Donaldson-Thomas invariants $D_{d, n}$ to Gromov-Witten invariants leads to

$$
Z_{D T}(q, t)=\sum_{d, n} D_{d, n} q^{n} \mathrm{e}^{-d t}=Z_{\mathrm{GV}}^{\prime}(-q, t) M(-q)^{\chi(X)}
$$

where

$$
M(q)=\prod_{n=1}^{\infty} \frac{1}{\left(1-q^{n}\right)^{n}}
$$

is the MacMahon function. This term reinstalls the constant map contribution. We list for reference a few Donaldson-Thomas invariants $D_{d, n}$ on the quintic in Table 4.1.

After an extensive discussion of possible tests of the OSV conjecture [37], the authors of [12] isolate as a crucial question for the validity of the latter the growth behaviour of 


\begin{tabular}{|r|rrrrrr|}
\hline $\mathrm{d} / \mathrm{n}$ & -3 & -2 & -1 & 0 & 1 & 2 \\
\hline 0 & 0 & 0 & 0 & 0 & 2875 & 569250 \\
1 & 0 & 0 & 0 & 0 & 609250 & 124762875 \\
2 & 0 & 0 & 0 & 609250 & 439056375 & 76438831000 \\
3 & 0 & 8625 & 2294250 & 4004590375 & 1010473893000 & 123236265797125 \\
\hline
\end{tabular}

Table 4.1: Donaldson-Thomas invariants.

the Donaldson-Thomas invariants. This behaviour is encoded in the scaling exponent $k$, defined as

$$
\log \left(D_{\lambda^{2} d, \lambda^{3} n}\right) \sim \lambda^{k} .
$$

The question is relevant in the range $d^{3}-n^{2}>0$ for which stable black hole configurations exist.

Because of Castelnouvo's bound, and since our data are up to genus 31, we can calculate the Donaldson-Thomas invariants exactly in the range $0 \leq d \leq 15$ and for arbitrary high $n$ for the quintic. We are interested in the limit

$$
k=\lim _{\lambda \rightarrow \infty} \frac{\log \log \left|D_{\lambda^{2} d, \lambda^{3} n}\right|}{\log \lambda} .
$$

In order to evaluate it for given values $(d, n)$ we chose $\lambda$ so that $d+l=\lambda^{2} d$ for $d, l \in \mathbb{N}$ and use the fact that $\log \left|D_{d+l, n}\right|$ for fixed $d, k$ scales in good approximation linearly with $n$ to calculate the interpolated value of the $D_{d+l, n^{\prime}}$ at $n^{\prime}=\lambda^{3}(d, l) n$, with $\lambda(d, l)=\sqrt{\frac{d+l}{d}}$. For $(d, 0)$ the latter interpolation is of course completely irrelevant and for charges for which the $n^{\prime}$ values become large it is not very relevant.

The leading correction to (4.8) is of order $1 /(\log (\lambda))$. It makes therefore sense to eliminate this leading correction by logarithmic Richardson-Thomas transforms. We define

$$
k_{l}^{(0)}=\frac{\log \log \left|D_{\lambda(d, l)^{2} d, \lambda(d, l)^{3} n}\right|}{\log \lambda(d, l)},
$$

and the $m$ th logarithmic Richardson-Thomas transform as

$$
k_{l}^{(m)}=\frac{k_{l+1}^{(m-1)} \log (l+1)-k_{l}^{(m-1)} \log (l)}{\log (l+1)-\log (l)} .
$$

With our knowledge of the topological string up to $g=31$ for the quintic we can evaluate the Donaldson-Thomas invariants up to degree 15. We plot in the first two graphs (5) the data for the $k_{l}^{(0)}$ and its first two logarithmic Richardson-Thomas transforms. The graphs clearly indicate that the convergence is improved by the transform. So even if there are subleading terms of other forms, we certainly managed to supress the leading correction and speed up the convergence. The data further show that there is an universal 

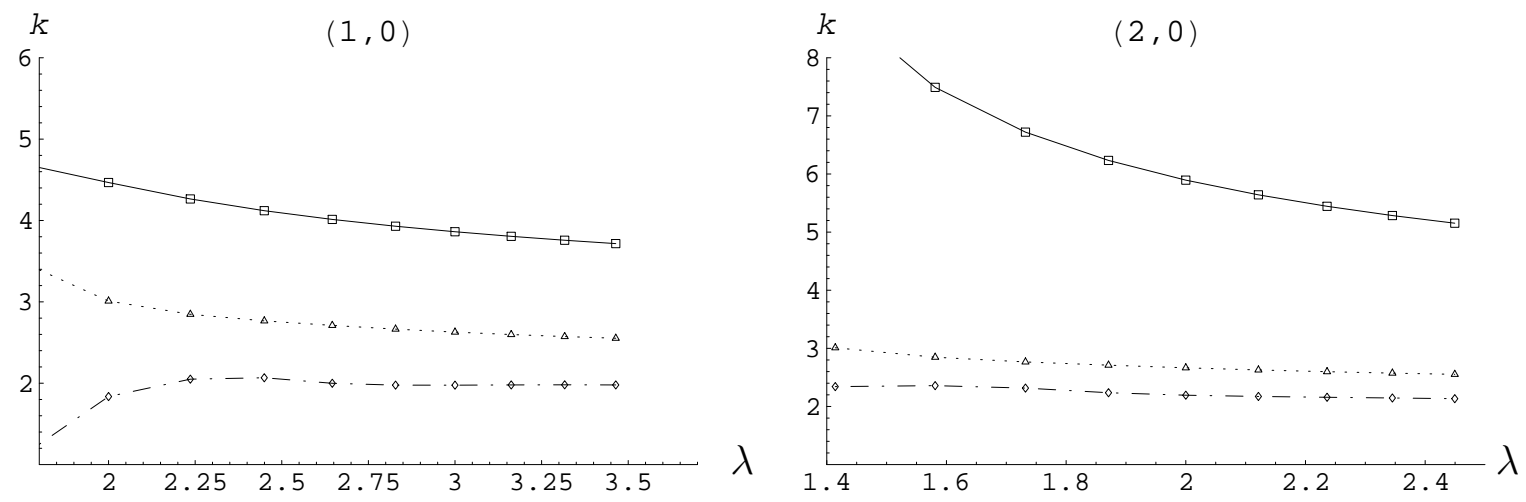

Figure 5: Scaling data $k^{(0)}(\square)$ and the transforms $k^{(1)}(\triangle), k^{(2)}(\diamond)$ for the DonaldsonThomas invariants on the quintic in $\mathbb{P}^{4}$ starting for $(d, 0)$ states.

behaviour independent of $d$ and that the value of $k$ is within the $2 \%$ range close to 2 . The higher logarithmic Richardson-Thomas transforms are consistent with this value but do not determine it better as we also have to take into account values with smaller $l$ hence smaller $\lambda$. We next test the universality of these results for other charges $(d, n)$ in Fig. 6. If $n \neq 0$ we need the interpolation for the $n^{\prime}$ values. This introduces some random subleading errors, which are of the order of the improvement by the second logarithmic RichardsonThomas transform. However as in the figure for $(2,0)$ we see that higher $d$ seems to lower the coefficient of the sub-sub-leading correction and makes already the second Richardson transform to converge reasonably well -well enough at least to conclude that the $k$ is considerably lower then 3 and very well compatible with the value $k=2 \pm 0.03$ found for the previous charges. We solved the bicubic in $\mathbb{P}^{5}$ up to genus 29 , which yields complete informations about the Donaldson-Thomas invariants up to degree 18. A similar analysis as above confirms the analysis for the quintic. The corresponding plots are in Fig. 7 and Fig. 8. Again a detailed summary of the data for more models can be found at [43]. We note a slight noise in the transform $k^{(1)}$ in Fig. 8, which is presumably due to the interpolation in the $n$ value of $D_{d, n}$ described above. The results for the other models are similar, but somewhat less precise due to smaller values of $d$ that are currently available.

To summarize: our analysis indicates that the value of $k$ is indeed universal and close to $k=2$. This strongly suggests that the "mysterious cancellations" [12 that eventually make possible to extend the the OSV conjecture to small coupling, actually take place. 

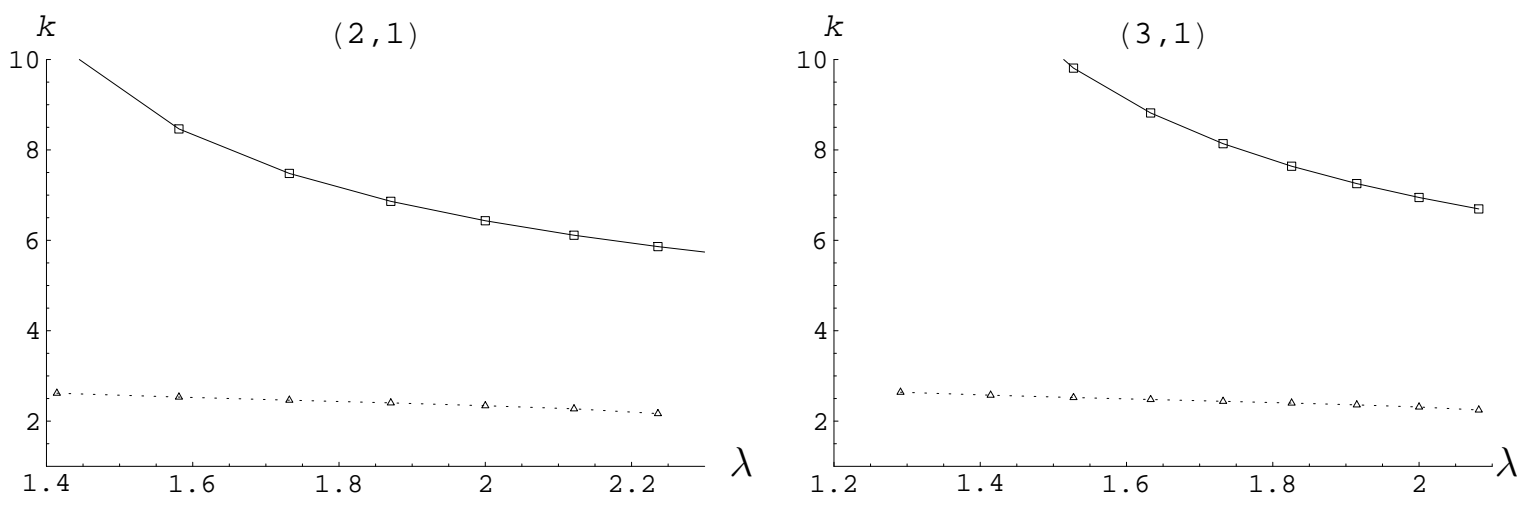

Figure 6: Scaling data $k^{(0)}(\square)$ and the first transform $k^{(1)}(\triangle)$ for the Donaldson-Thomas invariants on the quintic in $\mathbb{P}^{4}$ for the $(2,1)$ and $(3,1)$ states.
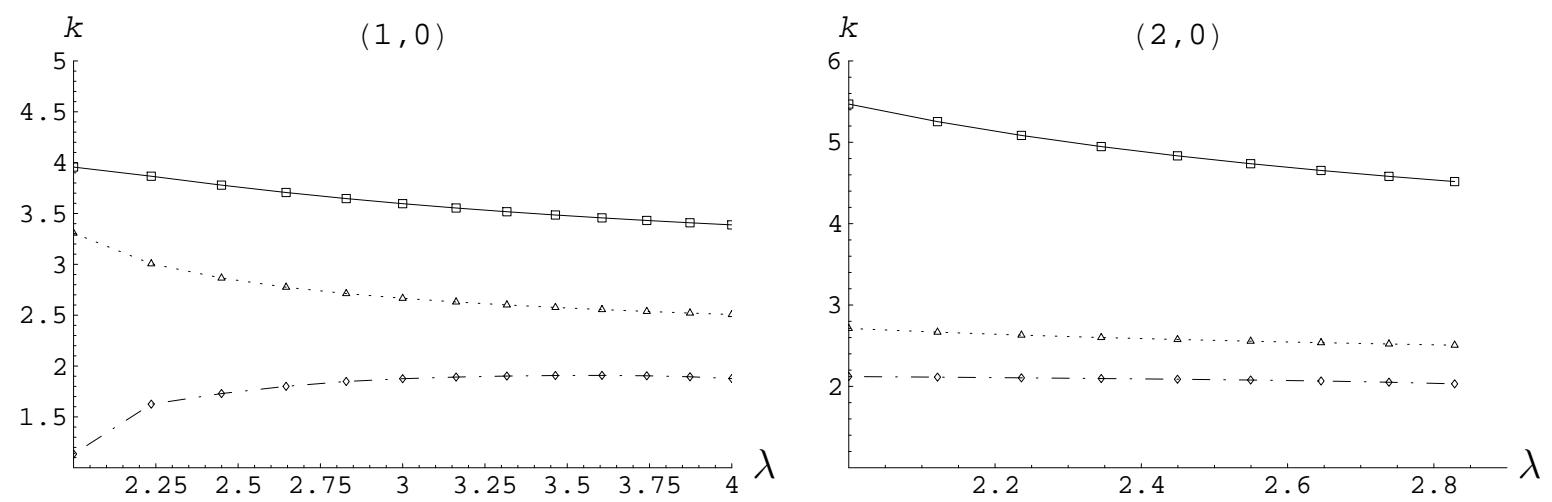

Figure 7: Scaling data $k^{(0)}(\square)$ and the transforms $k^{(1)}(\triangle), k^{(2)}(\diamond)$ for the DonaldsonThomas invariants on the bic-cubic complete intersection in $\mathbb{P}^{5}$ starting for $(d, 0)$ states.
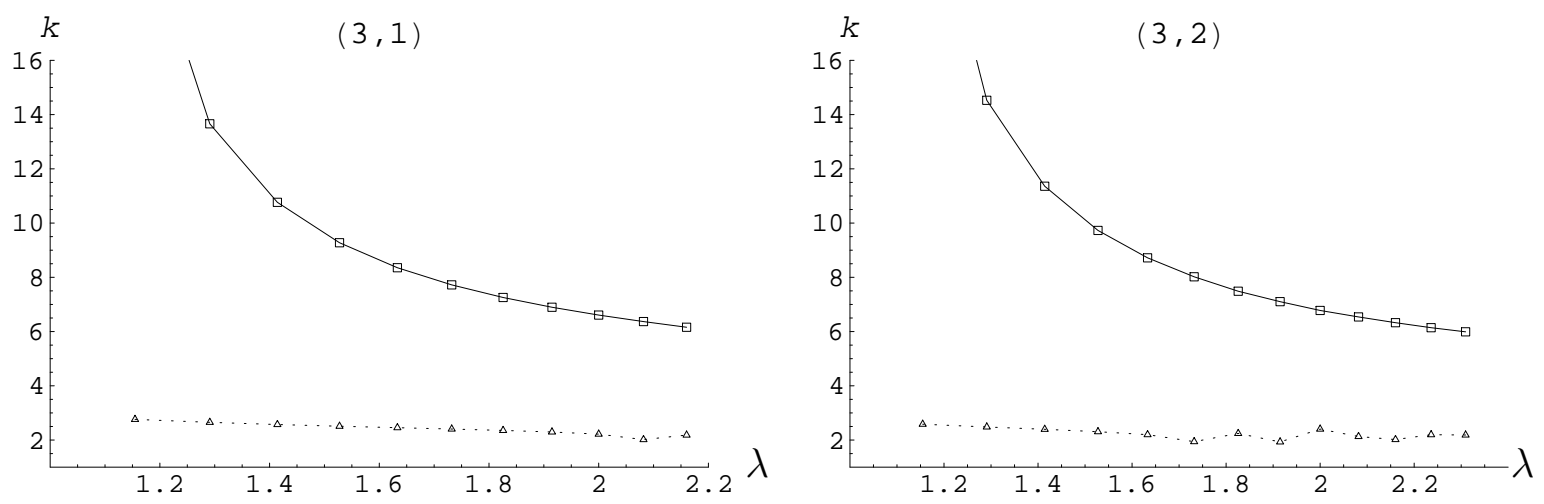

Figure 8: Scaling data $k^{(0)}(\square)$ and the first transform $k^{(1)}(\triangle)$ for the Donaldson-Thomas invariants on the bic-cubic complete intersection in $\mathbb{P}^{5}$ for the $(3,1)$ and $(3,2)$ states. 


\section{$5 \quad$ K3 fibrations}

\subsection{Topological strings on K3 fibrations}

We will now consider Calabi-Yau manifolds $X$ that have the structure of a K3 fibration, i.e. there is a fibration of the form

$$
\pi: X \rightarrow \mathbb{P}^{1}
$$

where the fibers are K3 surfaces. When the fibration is regular the homology of $X$ can be written as

$$
H_{2}(X, \mathbb{Z})=\left\langle\left[\mathbb{P}^{1}\right]\right\rangle \oplus \operatorname{Pic}(\mathrm{K} 3),
$$

where Pic(K3) is the Picard lattice of the K3 fiber. The rank of this lattice will be denoted by $\rho$, and $\Sigma^{a}, a=1, \cdots, \rho$ will denote a basis for this lattice. Let $\omega$ be the complexified Kähler form on $X$. The complexified Kähler parameters of $X$ are given by

$$
S=\int_{\mathbb{P}^{1}} \omega, \quad t^{a}=\int_{\Sigma^{a}} \omega, \quad a=1, \cdots, \rho .
$$

We will denote by $\eta_{S}, \eta_{a}$ the two-forms which are dual to $\mathbb{P}^{1}, \Sigma^{a}$.

It turns out that type IIA string theory compactified on these manifolds is very often dual to heterotic string theory compactified on $\mathrm{K} 3 \times \mathrm{T}^{2}$ [27, 31. Under this duality, $S$ becomes the axidilaton of the heterotic string. It follows that in the regime $S \rightarrow \infty$ one can map computations in the type IIA theory to perturbative computations in the heterotic string. In particular, the $F_{g}$ couplings of topological string theory (which are graviphoton couplings in type IIA theory) can be computed exactly at one-loop in the heterotic string, provided the Kähler parameters are restricted to the K3 fiber [27, 31, 25, 2, We will now review here some of these results.

The topological string amplitudes $F_{g}(S, t)$ on these fibrations have the following structure,

$$
\begin{aligned}
& F_{0}(S, t)=\frac{1}{6} C_{a b c} t^{a} t^{b} t^{c}+\frac{1}{2} C_{a b} S t^{a} t^{b}+\frac{\zeta(3)}{2} \chi(X)+\mathcal{F}_{0}(t)+\mathcal{O}\left(\mathrm{e}^{-S}\right), \\
& F_{1}(S, t)=\frac{1}{24}\left(c_{S} S+c_{a} t^{a}\right)+\mathcal{F}_{1}(t)+\mathcal{O}\left(\mathrm{e}^{-S}\right), \\
& F_{g}(S, t)=d_{g} \chi(X)+\mathcal{F}_{g}(t)+\mathcal{O}\left(\mathrm{e}^{-S}\right), \quad g \geq 2 .
\end{aligned}
$$

In these formulae, $C_{a b c}$ and $C_{a b}$ are triple intersection numbers in the fiber and in the mixed fiber/base direction, respectively. Notice that

$$
C_{a b c}=\int_{X} \eta_{a} \wedge \eta_{b} \wedge \eta_{c}, \quad C_{a b}=\int_{X} \eta_{S} \wedge \eta_{a} \wedge \eta_{b}
$$

We also have

$$
c_{a}=\int_{X} c_{2}(X) \wedge \eta_{a}, \quad a=1, \cdots, \rho, \quad c_{S}=\int c_{2}(X) \wedge \eta_{S} .
$$


For K3 fibrations with trivial fundamental group one has $c_{S}=24$ [36], but for the Enriques Calabi-Yau (which we will also analyze), $c_{S}=12$. The coefficient $d_{g}$ is the contribution of constant maps written down in (2.11). In (5.4), $\mathcal{F}_{g}(t)$ denotes the contribution of worldsheet instantons in the K3 fiber. It follows from [2, 25, 29, 34, 30] that the $\mathcal{F}_{g}(t)$ can be completely determined in terms of a single modular form that we will denote $f_{X}(q)$. In order to write down an explicit formula for $\mathcal{F}_{g}(t)$, we have to introduce the quasimodular forms $\mathcal{P}_{g}(q)$ which are defined by

$$
\left(\frac{2 \pi \eta^{3} \lambda}{\vartheta_{1}(\lambda \mid \tau)}\right)^{2}=\sum_{g=0}^{\infty}(2 \pi \lambda)^{2 g} \mathcal{P}_{g}(q) .
$$

The quantities $\mathcal{P}_{g}(q)$ can be explicitly written in terms of generalized Eisenstein series [34], and one has for example

$$
\mathcal{P}_{1}(q)=\frac{1}{12} E_{2}(q), \quad \mathcal{P}_{2}(q)=\frac{1}{1440}\left(5 E_{2}^{2}+E_{4}\right) .
$$

We now introduce the coefficients $c_{g}(n)$ through

$$
\mathcal{P}_{g}(q) f_{X}(q)=\sum_{n} c_{g}^{X}(n) q^{n}
$$

One then has the following expression for the heterotic $\mathcal{F}_{g}(t)$ :

$$
\mathcal{F}_{g}(t)=\sum_{Q \in \operatorname{Pic}(\mathrm{K} 3)} c_{g}^{X}\left(Q^{2} / 2\right) \operatorname{Li}_{3-2 g}\left(\mathrm{e}^{-Q \cdot t}\right),
$$

where $\mathrm{Li}_{n}$ is the polylogarithm of index $n$

$$
\operatorname{Li}_{n}(x)=\sum_{k=1}^{\infty} \frac{x^{k}}{k^{n}}
$$

In (5.10) we have also denoted

$$
Q \cdot t=n_{a} t^{a}, \quad Q^{2}=C^{a b} n_{a} n_{b},
$$

where $C^{a b}=C_{a b}^{-1}$ is the intersection form of the Picard lattice Pic(K3).

We will particularly interested in three special K3 fibrations: the STU model, the ST model, and the Enriques Calabi-Yau. Let us give some extra details for these cases:

- The STU model has $\rho=2$ and it can be realized by a complete intersection in a weighted projective space which is frequently denoted by $X_{24}(1,1,2,8,12)$. It has Euler characteristic $\chi=-480$. The classical prepotential can be obtained from the nonvanishing intersection numbers,

$$
C_{111}=8, \quad C_{112}=2, \quad C_{11}=2, \quad C_{12}=1,
$$


while the classical part of $F_{1}(S, t)$ is encoded by

$$
c_{1}=92, \quad c_{2}=c_{S}=24 .
$$

The modular form encoding the information about topological string amplitudes in the fiber is given by [34]

$$
f_{\mathrm{STU}}(q)=-\frac{2 E_{4} E_{6}}{\eta^{24}}(q) .
$$

It is sometimes useful to parametrize the Kähler cone in terms of the variables

$$
T=t_{1}+t_{2}, \quad U=t_{1},
$$

In this basis one has $Q^{2} / 2=m n$.

- The ST model has $\rho=1$ and is realized in type IIA by the CY $X_{12}(1,1,2,2,6)$. It has $\chi=-252$ and the classical intersection numbers

$$
C_{111}=4, \quad C_{11}=2,
$$

as well as

$$
c_{1}=52, \quad c_{S}=24 .
$$

The Kähler parameter along the fiber is usually denoted as

$$
T=t_{1}
$$

The relevant modular form is [29, 30]

$$
f_{\mathrm{ST}}(q)=-\frac{2 \theta E_{4} F_{6}}{\eta^{24}}(q),
$$

where

$$
\begin{aligned}
\theta(q) & =\sum_{n \in \mathbb{Z}} q^{\frac{n^{2}}{4}}=\vartheta_{3}(\tau / 2) \\
F_{2} & =\frac{1}{16} \vartheta_{2}^{4}(\tau / 2), \\
F_{6} & =E_{6}-2 F_{2}\left(\theta^{4}-2 F_{2}\right)\left(\theta^{4}-16 F_{2}\right) .
\end{aligned}
$$

Notice that $Q^{2} / 2=n^{2} / 4$.

- The Enriques Calabi-Yau is given by the free quotient $\left(\mathrm{K} 3 \times \mathbb{T}^{2}\right) / \mathbb{Z}_{2}$, and was introduced in the context of type II/heterotic duality in [18]. It is an elliptic fibration with $\rho=10$. It has $C_{a b c}=0$, while $C_{a b}$ is given by the intersection numbers of the Enriques surface $E$. The Picard lattice is

$$
\operatorname{Pic}(\mathrm{K} 3)=\Gamma^{1,1} \oplus E_{8}(-1),
$$


and

$$
c_{a}=0, \quad c_{S}=12 .
$$

The topological string amplitudes in the fiber were obtained in [32] (see also [22]). They are also controlled by a single modular form

$$
f_{E}(q)=-\frac{2}{\eta^{12}\left(q^{2}\right)},
$$

but their form is slightly different from (5.10)

$$
F_{g}(t)=\sum_{Q \in \operatorname{Pic}(\mathrm{K} 3)} c_{g}^{E}\left(Q^{2}\right)\left\{2^{3-2 g} \operatorname{Li}_{3-2 g}\left(\mathrm{e}^{-Q \cdot t}\right)-\mathrm{Li}_{3-2 g}\left(\mathrm{e}^{-2 Q \cdot t}\right)\right\},
$$

where $c_{g}^{E}(n)$ are defined again by (5.9) .

\subsection{Microscopic degeneracies and their asymptotic expansion}

We have seen that, at least in the case of topological strings on K3 fibrations, and for classes $Q$ restricted to the K3 fiber, one can obtain closed formula for the topological string amplitudes at all genera. It should be therefore possible to extract a closed formula for the generating functional of Gopakumar-Vafa invariants. In fact, by using the product formula

$$
\vartheta_{1}(\nu \mid \tau)=-2 q^{\frac{1}{8}} \sin (\pi \nu) \prod_{n=1}^{\infty}\left(1-q^{n}\right)\left(1-2 \cos (2 \pi \nu) q^{n}+q^{2 n}\right)
$$

one finds from the expression (5.10) and the structure (2.20)

$$
\sum_{Q \in \operatorname{Pic}(\mathrm{K} 3)} \sum_{r=0}^{\infty} n_{Q}^{r} z^{2 r} p^{Q^{2} / 2}=f_{X}(p) \xi^{2}(z)
$$

where $\xi(z)$ is the function that appears in helicity supertraces,

$$
\xi(z)=\prod_{n=1}^{\infty} \frac{\left(1-p^{n}\right)^{2}}{\left(1-p^{n}\right)^{2}+z^{2} p^{n}}=\prod_{n=1}^{\infty} \frac{\left(1-p^{n}\right)^{2}}{\left(1-p^{n} y\right)\left(1-p^{n} y^{-1}\right)},
$$

where we have set $z=-\mathrm{i}\left(y^{\frac{1}{2}}-y^{-\frac{1}{2}}\right)$.

We can now obtain a closed formula for the microscopic degeneracies. In order to have a description which incorporates as well the elliptic genus, we will count the microstates as in (2.18) but with $r \rightarrow r-1$. With this definition, the l.h.s. of (5.27), expanded in $q, y$, is precisely the generating function of microscopic degeneracies $\Omega(Q, m)$, summed over all $m, Q$. We then arrive to the expression

$$
\sum_{Q \in \operatorname{Pic}(\mathrm{K} 3)} \sum_{m=-\infty}^{\infty} \Omega(Q, m) y^{m} p^{Q^{2} / 2}=f_{X}(p) \xi^{2}(\nu, \sigma),
$$


where we have written

$$
y=\mathrm{e}^{2 \pi \mathrm{i} \nu}, \quad p=\mathrm{e}^{2 \pi \mathrm{i} \sigma} .
$$

Notice that if we consider $X=\mathrm{K} 3 \times \mathbb{T}^{2}$ and restrict to classes $Q$ in the fiber, the counting of microstates given by the elliptic genus is

$$
\chi\left(S_{p} \mathrm{~K} 3 ; q, y\right)_{q^{0}}=\prod_{N=1}^{\infty} \frac{1}{\left(1-p^{N}\right)^{20}\left(1-p^{N} y\right)^{2}\left(1-p^{N} y^{-1}\right)^{2}}=\frac{p}{\eta^{24}(p)} \xi^{2}(y) .
$$

This has the same form than (5.29) with

$$
f_{\mathrm{K} 3 \times T^{2}}(p)=\frac{1}{\eta^{24}(p)},
$$

therefore we can consider the "small" D1-D5 system as a particular case of our analysis.

The expression (5.29) tells us that the microscopic degeneracies we are looking for are simply the Fourier coefficients of the object in the r.hs. We can then invert it to write

$$
\Omega(N, m)=\int_{-\frac{1}{2}+\mathrm{i} 0^{+}}^{\frac{1}{2}+\mathrm{i} 0^{+}} \mathrm{d} \sigma \int_{0}^{1} \mathrm{~d} \nu \mathrm{e}^{-2 \pi \mathrm{i}(N \sigma+m \nu)} \Phi(\nu, \sigma), \quad N=Q^{2} / 2,
$$

where we defined

$$
\Phi(\nu, \sigma)=f_{X}(p) \xi^{2}(\nu, \sigma) .
$$

and we have assumed that $N$ is a non-negative integer (this can be guaranteed by rescaling $p \rightarrow p^{k}$ for some appropriate $k$ ). The contour in (15.33) has been chosen to avoid the poles in the integrand.

We will now evalute the asymptotic expansion of $\Omega(N) \equiv \Omega(N, 0)$ in inverse powers of $N$. Nonzero values of the spin $m=0$ can be analyzed in a similar way. The expression we will find is exact up to corrections which are exponentially suppressed in the large charge limit $N \rightarrow \infty$. Notice that in our situation we can not appeal to the Rademacher expansion which was used in [15, 9], since (5.34) is not a Jacobi form (it can be regarded as a Jacobi form with negative index). It is likely that an analog of the Rademacher expansion exists, but we will perform a direct evaluation of the integral (5.33) in the spirit of the counting of states with spin in Appendix $\mathrm{C}$ of [9] and in [10].

First of all, we reexpress the integrand (5.34) in terms of $\vartheta_{1}(\nu \mid \sigma)$ as,

$$
\Phi(\nu, \sigma)=4 \sin ^{2}(\pi \nu) \eta^{6}(p) \frac{f_{X}(p)}{\vartheta_{1}^{2}(\nu \mid \sigma)} .
$$

Using the modular behavior of $\vartheta_{1}(\nu \mid \sigma)$ under the $S$ transformation $\sigma \rightarrow \tilde{\sigma}=-1 / \sigma$ we get,

$$
\vartheta_{1}(\nu, \sigma)=-\frac{2 \mathrm{i}}{\sqrt{-\mathrm{i} \sigma}} \mathrm{e}^{\frac{\pi}{\mathrm{i} \sigma}\left(\nu^{2}+\frac{1}{4}\right)} \sin \left(\frac{\pi \nu}{\sigma}\right)\left\{1+O\left(\mathrm{e}^{-\frac{2 \pi \mathrm{i}}{\sigma}}\right)\right\} .
$$


It is easy to see that the saddle point evaluation of (5.33) is governed by

$$
\sigma_{*}=\frac{\mathrm{i}}{\sqrt{N}}+\mathcal{O}\left(\frac{1}{N}\right)
$$

Therefore, the corrections to (5.36) will be exponentially suppressed. Using the modularity of $\eta(p)$, and taking the part of the sin in the denominator which is not exponentially suppressed, we obtain,

$$
\Phi(\nu, \sigma) \sim-4 \sigma^{-2} \mathrm{e}^{2 \frac{\mathrm{i} \pi}{\sigma}\left(\nu^{2}-\nu\right)} \sin ^{2}(\pi \nu) f_{X}(p) .
$$

Therefore, in order to compute the asymptotics of (5.33) we just need

$$
\Omega(N) \sim-4 \int_{-\frac{1}{2}+\mathrm{i} 0^{+}}^{\frac{1}{2}+\mathrm{i} 0^{+}} \mathrm{d} \sigma \mathrm{e}^{-2 \pi \mathrm{i} N \sigma} \frac{f_{X}(p)}{\sigma^{2}} \int_{0}^{1} \mathrm{~d} \nu \mathrm{e}^{2 \frac{\mathrm{i} \pi}{\sigma}\left(\nu^{2}-\nu\right)} \sin ^{2}(\pi \nu) .
$$

The integral over $\nu$ is easily worked out in terms of the error function $\operatorname{Erf}(x)$, as follows,

$$
\begin{aligned}
& \int_{0}^{1} \mathrm{~d} \nu \mathrm{e}^{2 \frac{\mathrm{i} \pi}{\sigma}\left(\nu^{2}-\nu\right)} \sin ^{2}(\pi \nu)=\sqrt{\frac{\mathrm{i} \sigma}{8}} \mathrm{e}^{\frac{\pi}{2 \mathrm{i} \sigma}} \operatorname{Erf}\left(\sqrt{\frac{\pi}{2 \mathrm{i} \sigma}}\right) \\
& +\sqrt{\frac{\mathrm{i} \sigma}{32}} \mathrm{e}^{\frac{\pi}{2 \mathrm{i}}\left(\sigma+\frac{1}{\sigma}\right)}\left\{\operatorname{Erf}\left(\sqrt{\frac{\pi}{2 \mathrm{i} \sigma}}(\sigma+1)\right)-\operatorname{Erf}\left(\sqrt{\frac{\pi}{2 \mathrm{i} \sigma}}(\sigma-1)\right)\right\} .
\end{aligned}
$$

Due to (5.37) we can use the asymptotic expansion of the Erf function,

$$
\operatorname{Erf}(x) \sim 1-\frac{\mathrm{e}^{-x^{2}}}{\sqrt{\pi}} \sum_{r=0}^{\infty}(-1)^{r} \frac{(2 r-1) ! !}{2^{r}} x^{-(2 r+1)}, \quad|x| \rightarrow \infty,|\arg (-x)|<\pi .
$$

Ignoring terms which are exponentially suppressed at large $N$, we find,

$$
\int_{0}^{1} \mathrm{~d} \nu \mathrm{e}^{2 \frac{\mathrm{i} \pi}{\sigma}\left(\nu^{2}-\nu\right)} \sin ^{2}(\pi \nu) \sim-\frac{1}{4} \sum_{r=0}^{\infty} \frac{\mathrm{i}^{1+3 r}}{\pi^{1+r}}(2 r-1) ! ! G_{r}(\sigma),
$$

with,

$$
G_{r}(\sigma)=\sigma^{r+1}\left(2+\frac{1}{(\sigma-1)^{1+2 r}}-\frac{1}{(\sigma+1)^{1+2 r}}\right) .
$$

Again, due to (5.37), we can expand it around $\sigma=0$,

$$
G_{r}(\sigma)=-2 \sum_{s=0}^{\infty}\left(\begin{array}{c}
2(1+s+r) \\
2 r
\end{array}\right) \sigma^{3+2 s+r} .
$$

Putting all together, we obtain,

$$
\Omega(N) \sim 2 \sum_{r=0}^{\infty} \frac{(2 r-1) ! !}{(\mathrm{i} \pi)^{r+1}} \sum_{s=0}^{\infty}\left(\begin{array}{c}
2(1+s+r) \\
2 r
\end{array}\right) \int_{-\frac{1}{2}+\mathrm{i} 0^{+}}^{\frac{1}{2}+\mathrm{i} 0^{+}} \mathrm{d} \sigma \mathrm{e}^{-2 \pi \mathrm{i} N \sigma} f_{X}(p) \sigma^{1+2 s+r} .
$$


We now work out the integral,

$$
A_{s, r}(N) \equiv \int_{-\frac{1}{2}+\mathrm{i} 0^{+}}^{\frac{1}{2}+\mathrm{i} 0^{+}} \mathrm{d} \sigma \mathrm{e}^{-2 \pi \mathrm{i} N \sigma} f_{X}(p) \sigma^{1+2 s+r}
$$

We assume that $f_{X}(p)$ has modular weight $w$, so that $f_{X}(p)=\sigma^{-w} f_{X}(\tilde{p})$, where $\tilde{p}=\mathrm{e}^{-\frac{2 \pi \mathrm{i}}{\sigma}}$. For the modular forms that we consider here, $f_{X}(\tilde{p})=c \tilde{p}^{-\alpha}+\cdots$, and the integral above gives a modified Bessel function

$$
A_{s, r}(N) \sim c \mathrm{i}^{1+2 s+r-w} \hat{I}_{2 s+r+2-w}(4 \pi \sqrt{\alpha N}) .
$$

We end up then with the following result for the exact asymptotics of the microscopic black hole degeneracy,

$$
\Omega(N) \sim 2 c \mathrm{i}^{w} \sum_{r=0}^{\infty} \frac{(2 r-1) ! !}{\pi^{r+1}} \sum_{s=0}^{\infty}(-1)^{s}\left(\begin{array}{c}
2(1+s+r) \\
2 r
\end{array}\right) \hat{I}_{2 s+r+2-w}(4 \pi \sqrt{\alpha N}) .
$$

Using now the formula for the asymptotic expansion of $\hat{I}$ functions (see for example App. A of [9]), we find for the entropy $S(N)=\log \Omega(N)$ the following expansion

$$
S \sim 4 \pi \sqrt{\alpha N}-\frac{5-2 w}{4} \log (N)+\log \left(\frac{\sqrt{2} \mathrm{i}^{w} \alpha^{\frac{2 w-5}{4}} c}{\pi}\right)+\frac{177+16 w-4 w^{2}}{32 \pi \sqrt{\alpha}} \frac{1}{\sqrt{N}}+\mathcal{O}\left(N^{-1}\right) .
$$

The expansion in powers of $1 / N^{\frac{1}{2}}$ in (5.48), which is obtained by using the asymptotics of modified Bessel functions, is the expansion of the original integral around the saddle point (5.37). This can be verified by an explicit computation of the first few orders of the saddlepoint expansion.

Let us now evaluate (5.49) in some examples. For $\mathrm{K} 3 \times \mathbb{T}^{2}$ we have $(w, \alpha, c)=$ $(-12,1,1)$, and the entropy reads

$$
S \sim 4 \pi \sqrt{N}-\frac{29}{4} \log (N)+\log \left(\frac{\sqrt{2}}{\pi}\right)-\frac{591}{32 \pi} \frac{1}{\sqrt{N}}+\mathcal{O}\left(N^{-1}\right) .
$$

For the STU model, with the values $(w, \alpha, c)=(-2,1,-2)$, we find

$$
S \sim 4 \pi \sqrt{N}-\frac{9}{4} \log (N)+\log \left(\frac{\sqrt{8}}{\pi}\right)+\frac{129}{32 \pi} \frac{1}{\sqrt{N}}+\mathcal{O}\left(N^{-1}\right) .
$$

The ST model is slightly different, since in $f_{\mathrm{ST}}(p)$ both integer and rational powers of $p$ appear. As mentioned above, we should redefine $p \rightarrow p^{4}$ and write down the generating functional for the degeneracies as

$$
\sum_{Q \in \operatorname{Pic}(\mathrm{K} 3)} \sum_{m=-\infty}^{\infty} \Omega(Q, m) y^{m} p^{2 Q^{2}}=f_{\mathrm{ST}}\left(p^{4}\right) \xi^{2}(\nu, 4 \sigma),
$$


where we recall that $M \equiv 2 Q^{2}=n^{2}$ is an integer. The asymptotics is given by the integral

$$
\Omega_{\mathrm{ST}}(M) \sim-\int_{-\frac{1}{2}+\mathrm{i} 0^{+}}^{\frac{1}{2}+\mathrm{i} 0^{+}} \mathrm{d} \sigma \mathrm{e}^{-2 \pi \mathrm{i} M \sigma} \frac{f_{\mathrm{ST}}\left(p^{4}\right)}{4 \sigma^{2}} \int_{0}^{1} \mathrm{~d} \nu \sin ^{2}(\pi \nu) \mathrm{e}^{\frac{\mathrm{i} \pi}{2 \sigma}\left(\nu^{2}-\nu\right)} .
$$

The integral over $\nu$ is given by (5.40) upon replacing $\sigma \rightarrow 4 \sigma$. Since,

$$
f_{\mathrm{ST}}\left(p^{4}\right)=-2 \frac{E_{4}\left(p^{4}\right) F_{6}\left(p^{4}\right)}{\eta^{24}\left(p^{4}\right)} \vartheta_{3}(2 \sigma) \sim-16 \sqrt{2 \mathrm{i}} \sigma^{\frac{3}{2}} \mathrm{e}^{\frac{\mathrm{i} \pi}{2 \sigma}},
$$

one finds in the end,

$$
\Omega_{\mathrm{ST}}(M) \sim \sqrt{2} \sum_{r=0}^{\infty} \frac{(2 r-1) ! !}{\pi^{r+1}} \sum_{s=0}^{\infty}(-1)^{s}\left(\begin{array}{c}
2(1+s+r) \\
2 r
\end{array}\right) \hat{I}_{\frac{7}{2}+2 s+r}(2 \pi \sqrt{M}),
$$

and from here one can read the entropy,

$$
S(Q) \sim 4 \pi \sqrt{\frac{1}{2} Q^{2}}-2 \log \left(Q^{2}\right)+\cdots
$$

Finally we turn to the case of Enriques CY manifold. It follows from (5.25) that one has to distinguish two types of homology classes: the classes $Q$ whose entries contain at least an odd integer (which were called odd classes in [32]), and the classes $Q$ for which all entries are even (called even classes). A simple calculation shows that the generating function of Gopakumar-Vafa invariants for the odd classes is given by

$$
\sum_{r=0}^{\infty} \sum_{Q \text { odd }} n_{Q}^{r} p^{Q^{2}} z^{r-1}=\frac{f_{E}(q)}{4 \sin ^{2}\left(\frac{\pi \nu}{2}\right)}\left(\xi^{2}(\nu / 2, p)-\xi^{2}(\nu / 2,-p)\right) .
$$

while for the even classes is given by

$$
\begin{aligned}
\sum_{r=0}^{\infty} \sum_{Q \text { even }} n_{Q}^{r} p^{Q^{2}} z^{r-1} & =\frac{f_{E}(q)}{4 \sin ^{2}\left(\frac{\pi \nu}{2}\right)}\left(\xi^{2}(\nu / 2, p)-\xi^{2}(\nu / 2,-p)\right) \\
& -f_{E}\left(q^{4}\right)\left(\xi^{2}\left(\nu, p^{4}\right)-\xi^{2}\left(\nu,-p^{4}\right)\right) .
\end{aligned}
$$

Notice that for even classes $Q^{2} \equiv 0 \bmod 4$, while for odd classes one only has $Q^{2} \equiv 0$ mod 2. In contrast to the previous K3 fibrations, in the above generating function we have $p^{Q^{2}}$, instead of $p^{Q^{2} / 2}$, and this will lead to a different leading term as compared for example to the STU model.

The computation of the asymptotics of the microstates is similar to the one that we just performed. Let us begin with odd classes. Using the identity,

$$
\xi^{2}(\nu,-p)=4 \sin ^{2}(\pi \nu) \frac{\eta^{6}(2 \sigma) \vartheta_{3}^{2}(2 \sigma)}{\vartheta_{1}^{2}(\nu \mid 2 \sigma) \vartheta_{3}^{2}(\nu \mid 2 \sigma)},
$$


and proceeding as in the previous case, we find,

$$
\Omega_{\text {odd }}(N)=\Omega_{1}(N)+\Omega_{2}(N), \quad N=Q^{2} / 2,
$$

where,

$$
\begin{aligned}
& \Omega_{1}(N) \sim 16 \int_{-\frac{1}{2}+\mathrm{i} 0^{+}}^{\frac{1}{2}+\mathrm{i} 0^{+}} \mathrm{d} \sigma \mathrm{e}^{-4 \pi \mathrm{i} N \sigma} \sigma^{2} \eta^{6}(2 \sigma) \vartheta_{3}^{2}(2 \sigma) f_{E}(p) \int_{0}^{1} \mathrm{~d} \nu \sin ^{2}(\pi \nu) \mathrm{e}^{\frac{\mathrm{i} \pi}{2 \sigma}\left(\nu^{2}-\nu+\frac{1}{2}\right)}, \\
& \Omega_{2}(N) \sim-4 \mathrm{i} \int_{-\frac{1}{2}+\mathrm{i} 0^{+}}^{\frac{1}{2}+\mathrm{i} 0^{+}} \mathrm{d} \sigma \mathrm{e}^{-4 \pi \mathrm{i} N \sigma} \sigma \eta^{6}(\sigma) f_{E}(p) \int_{0}^{1} \mathrm{~d} \nu \sin ^{2}(\pi \nu) \mathrm{e}^{\frac{\mathrm{i} \pi}{2 \sigma}(\nu-1)^{2}} .
\end{aligned}
$$

As before, we evaluate the integrals over $\nu$ in terms of the Erf function and its asymptotic expansion. We then use the modularity properties of the different functions involved here to obtain,

$$
\Omega_{\text {odd }}(N) \sim \frac{1}{16} \sum_{r=0}^{\infty} \frac{(2 r-1) ! !}{\pi^{r+1}} \sum_{s=0}^{\infty}(-1)^{s}\left(\begin{array}{c}
2(1+s+r) \\
2 r
\end{array}\right)\left(1-4^{-(1+r+s)}\right) \hat{I}_{8+2 s+r}(\pi \sqrt{8 N}) .
$$

Let us now consider the even classes, (5.58). Comparing (5.58) with (5.57), we see that,

$$
\Omega_{\text {even }}(N)=\Omega_{\text {odd }}(N)-\widetilde{\Omega}(N)
$$

where,

$$
\tilde{\Omega}(N)=\int_{-\frac{1}{2}+\mathrm{i} 0^{+}}^{\frac{1}{2}+\mathrm{i} 0^{+}} \mathrm{d} \sigma \int_{0}^{1} \mathrm{~d} \nu \mathrm{e}^{-4 \mathrm{i} \pi N \sigma} 4 \sin ^{2}(\pi \nu) f_{E}\left(p^{4}\right)\left(\xi^{2}\left(\nu, p^{4}\right)-\xi^{2}\left(\nu,-p^{4}\right)\right) .
$$

A computation similar to the one we performed shows that $\widetilde{\Omega}(N)$ is exponentially suppressed with respect to $\Omega_{\text {odd }}(N)$, since it leads to terms that go like $\exp (\pi \sqrt{2 N})$ and $\exp (\pi \sqrt{6 N})$. Therefore, as an asymptotic expansion in $1 / \sqrt{N}, \Omega_{\text {even }}(N) \sim \Omega_{\text {odd }}(N)$, and the asymptotics does not distinguish between the even and the odd classes. We finally obtain, for the small Enriques black hole,

$$
S_{E}(Q) \sim 2 \pi \sqrt{Q^{2}}-\frac{17}{2} \log \sqrt{Q^{2}}+\cdots
$$

The main conclusion of our analysis is that, in all cases, the leading term of the microscopic entropy for these black holes is given by

$$
S(Q) \sim 2 \pi \sqrt{\frac{c_{S}}{12} Q^{2}}
$$

since $c_{S}=24$ for $\mathrm{K} 3 \times \mathbb{T}^{2}$, the STU and the ST models, but $c_{S}=12$ for the Enriques CY. Of course, our analysis has also given precise formulae for the subleading terms. 
The leading behavior (5.66) can be also verified by a numerical analysis similar to the one performed in sections 3 and 4. For example, for the STU model we have computed the quantity $f(N)=S(N) / \sqrt{N}$ for $1 \leq N<50$, where $S(N)=\log \Omega(N)$. In order to subtract the logarithmic term in the asymptotic expansion (5.51) we consider the transform,

$$
A(N)=\frac{(N+1) S(N+2)-(2 N+1) S(N+1)+N S(N)}{(N+1) \sqrt{N+2}-(2 N+1) \sqrt{N+1}+N \sqrt{N}} .
$$

In Fig. 9 we plot $f(N)$ (bottom) and $A(N)$ (top). The horizontal line is the expected asymptotic value $4 \pi$ for both quantities as $N \rightarrow \infty$. As before, the transform $A(N)$ improves rapidly the convergence.

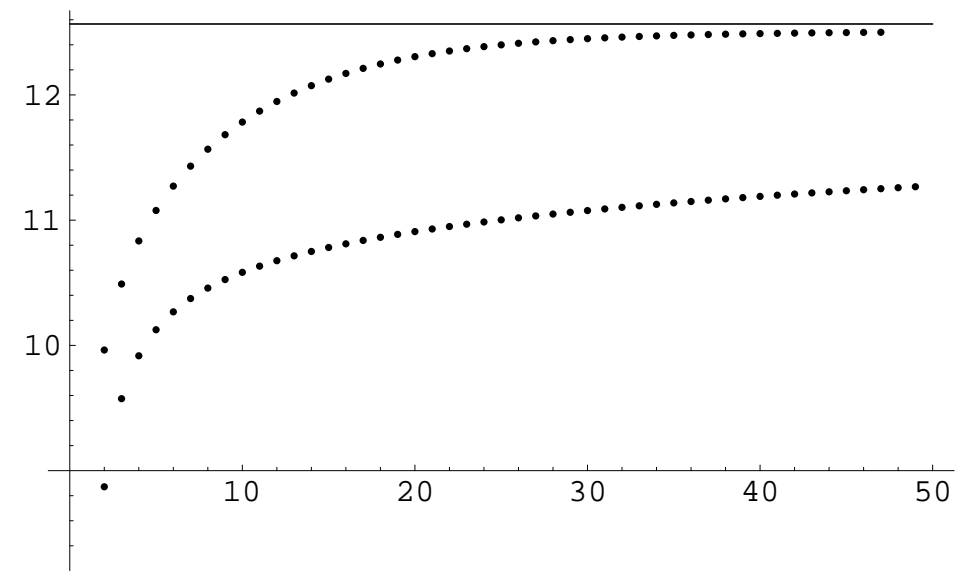

Figure 9: Microscopic data for $f(N)=S(N) / \sqrt{N}$ (bottom) and its transform $A(N)$ (top), defined in (5.67), for the STU model, and for $1 \leq N<50$. The horizontal line is the expected asymptotic value $4 \pi$.

\subsection{Macroscopic entropy for small black holes}

The $5 \mathrm{~d}$ black holes obtained by wrapping the M2 branes along cycles in the K3 fiber have actually vanishing classical entropy and are therefore small black holes. Indeed, as we have seen, the leading asymptotic degeneracy scales like $Q$, and not like $Q^{3 / 2}$. This is also what is found for small $4 \mathrm{~d}$ black holes [9].

Let us briefly show that the classical area of these black holes is zero for any set of intersection numbers $C_{a b c}, C_{a b}$. In order to do this, we can use the $5 \mathrm{~d}$ attractor mechanism described in section 2. Equivalently, by using the $4 \mathrm{~d} / 5 \mathrm{~d}$ connection of [20], we can map the $5 \mathrm{~d}$ black hole to a $4 \mathrm{~d}$ black hole with D6 charge $p^{0}=1$ and D2 charges $Q_{A}$. At the level of the leading macroscopic entropy, the $4 \mathrm{~d}$ computation gives the same result as the 
$5 \mathrm{~d}$ computation [20]. In the $4 \mathrm{~d}$ language, we start with the tree level SUGRA prepotential

$$
F=-\frac{1}{2} C_{a b} \frac{X^{S} X^{a} X^{b}}{X^{0}}-\frac{1}{6} C_{a b c} \frac{X^{a} X^{b} X^{c}}{X^{0}}
$$

We will do the computation for a generic D6-D2 charge, i.e. we will start with generic charges $p^{0}, Q_{a}, Q_{S}$, and then take the charge $Q_{S} \rightarrow 0$ at the end of the computation (as well as setting $p^{0}=1$ ). This will guarantee that we obtain generic solutions to the attractor mechanism.

Let us first assume that $C_{a b c}=0$, as it happens in $\mathrm{K} 3 \times \mathrm{T}^{2}$ and the Enriques CalabiYau. In this case, the attractor equations are easily solved as,

$$
\left(X_{*}^{0}, X_{*}^{S}, X_{*}^{a}\right)=\left(p^{0}, \mathrm{i} \sqrt{\frac{p^{0} Q^{2}}{2 Q_{S}}}, \mathrm{i} \sqrt{\frac{2 Q_{S} p^{0}}{Q^{2}}} Q^{a}\right),
$$

where

$$
Q^{2}=C^{a b} Q_{a} Q_{b}, \quad Q^{a}=C^{a b} Q_{b}
$$

The entropy is given by

$$
S=\pi \sqrt{2 p^{0} Q_{S} Q^{2}}
$$

and it vanishes in the limit $Q_{S} \rightarrow 0$. This is as expected.

If we now consider a general prepotential with nonvanishing $C_{a b c}$, the attractor equations are now solved at

$$
\left(X^{0}, X^{S}, X^{a}\right)=\left(p^{0}, \mathrm{i} \sqrt{\frac{p^{0}}{2 Q_{S}}} \xi^{S}, \mathrm{i} \sqrt{2 p^{0} Q_{S}} \xi^{a}\right)
$$

where the $\xi^{A}$ are solutions to,

$$
\begin{aligned}
\xi^{a} \xi_{a} & =1, \\
Q^{a} & =\xi^{S} \xi^{a}+Q_{S} C^{a b} C_{b e f} \xi^{e} \xi^{f} .
\end{aligned}
$$

Notice that, in these variables, the model with $C_{a b c}=0$ corresponds to the smooth values,

$$
\left(\xi_{*}^{S}, \xi_{*}^{a}\right)=\left(\sqrt{Q^{2}}, \frac{Q^{a}}{\sqrt{Q^{2}}}\right) .
$$

We can already see that, in the limit $Q_{S} \rightarrow 0$, the perturbation by $C_{a b c}$ in (5.73) vanishes, therefore in the limit of zero charge in the base the presence of nontrivial intersection numbers in the fiber should be unimportant. More formally, it is easy to see that one can construct a consistent solution of (5.73) of the form,

$$
\xi^{A}=\xi_{*}^{A}+\sum_{n=1}^{\infty} c_{n}^{A} Q_{S}^{n}
$$


where the coefficients $c_{n}^{A}$ depend on $C_{a b c}$ and can be calculated order by order. In terms of the $\xi^{A}$ the macroscopic entropy is

$$
S=\pi \sqrt{2 p^{0} Q_{S}}\left(C_{a b} \xi^{a} \xi^{b} \xi^{S}+\frac{2}{3} Q_{S} C_{a b c} \xi^{a} \xi^{b} \xi^{c}\right)
$$

and, in the limit $Q_{S} \rightarrow 0$, it will vanish irrespectively of the value of $C_{a b c}$. Therefore, 5 d black holes whose membrane charge is restricted to the K3 fiber of a K3 fibration are always small. This can be checked as well by detailed computations in different models (like the STU and ST models considered above).

Since the leading contribution to the entropy vanishes we should now look at the subleading terms in the macroscopic entropy. As we explained in section 2, it was shown in [23, 1, 8] that these terms are obtained by performing the shift

$$
Q_{A} \rightarrow \widehat{Q}_{A}=Q_{A}+\zeta c_{2 A}, \quad \zeta=\frac{1}{8}
$$

The leading term in the entropy for the small $5 \mathrm{~d}$ black hole is given (for large charge $Q$ ) by performing this shift in (5.71)

$$
S=2 \pi \sqrt{\frac{\zeta c_{S}}{2} Q^{2}} .
$$

This can be derived in detail by solving the attractor equations with shifted charges (5.77) as a power series in $1 / Q$, and then taking the limit $Q_{S} \rightarrow 0$. Notice that the entropy (5.78) only depends on $C_{a b}$ and $c_{S}$. Also, in this regime, the solutions of the attractor equations occur at values of the Kähler parameters which are of the order of the string size, and the SUGRA calculation might be problematic. Indeed, it is easy to see that (5.78) does not agree with the leading term of the asymptotics that we obtained in the previous subsection. By comparing (5.66) with (5.78) we find that the formula agree if we set instead $\zeta=1 / 6$. This is the value of $\zeta$ that is predicted by the $4 \mathrm{~d} / 5 \mathrm{~d}$ connection of [20].

In [23, 8] it was noticed that the subleading correction (5.77) obtained in a macroscopic $5 \mathrm{~d}$ computation was not in accord with the subleading correction predicted by [20] and the $4 \mathrm{~d}$ attractor mechanism. We now find that, for big $5 \mathrm{~d}$ black holes, the subleading correction for the microscopic entropy is in rough agreement with (5.77), while for small $5 \mathrm{~d}$ black holes the leading asymptotics is in accord with a $4 \mathrm{~d}$ computation for a small D6/D2 system with $p^{0}=1$. As we already mentioned, in the case of small black holes, the SUGRA computations with which we are comparing our results should receive large corrections, but in other situations they still lead to results which are in agreement with the microscopic counting, as in [9, 13. In our case we obtain a result in disagreement with the $5 \mathrm{~d}$ computation but in agreement with the $4 \mathrm{~d}$ computation. It would be interesting to resolve this puzzle. 


\section{Conclusions}

In this paper we have studied the microscopic counting of $5 \mathrm{~d}$ black hole states by using topological string theory. In the case of big black holes, we have given convincing numerical evidence that the BPS invariants encoded in the topological string amplitudes account correctly for the macroscopic entropy of spinning black holes. Moreover, we have also shown that the data favour the "mysterious cancellation" of [12] that makes possible to extend the validity of the OSV conjecture, and we were able to explore new aspects of black hole entropy which have not been studied before using supergravity. Clearly, it would be very desirable to improve our numerical results with more data. Using the interplay between modularity and an-holomophicity in topological string theory [42, 26, 22], analytic results on the asymtotics might be not out of reach 4 .

We also gave exact formulae for microscopic degeneracies of a class of small $5 \mathrm{~d}$ black holes, which are obtained by wrapping M2 branes in the fiber of a K3 fibration, and we computed the asymptotic expansion in inverse powers of the charge. As expected, the calculation shows that for small black holes the leading term in the entropy scales like $S \rightarrow \lambda S$ when the charges are scaled with $\lambda$. We found however that the coefficient of the leading term does not agree with the shift of charges obtained in [23, 1, 8] in a $5 \mathrm{~d}$ SUGRA computation. In principle there is no reason why these two computations should agree, since small $\mathcal{N}=1$ black holes are generically beyond the SUGRA approximation. On the other hand, the microscopic results are well reproduced by the $4 \mathrm{~d} / 5 \mathrm{~d}$ connection of [20] and a $4 \mathrm{~d}$ attractor computation. We should emphasize however that for big black holes the $5 \mathrm{~d}$ shift (5.77) fits our data better than the $4 \mathrm{~d}$ shift with $\zeta=1 / 6$. It would be very interesting to understand this better.

\section{Acknowledgments}

It is a pleasure to thank Davide Gaiotto, Thomas Grimm, Aki Hashimoto, Sheldon Katz, Wei Li, Boris Pioline, Nick Warner and Xi Yin for helpful discussions, and Frederik Denef for a very useful correspondence. We would like to thank as well Gregory Moore and Cumrun Vafa for their comments on the manuscript. Many thanks also to Max Kreuzer for generously granting us computer time. This work is partially supported by the DOE grant DE-F602-95ER40896. AT is supported by a Marie Curie fellowship.

\section{A General features of the instanton expansion}

The asymptotic behaviour at the conifold, Castelnouvo's theory, and the calculation via degenerate Jacobians, suggest some general features of the Gopakumar-Vafa expansion.

\footnotetext{
${ }^{4}$ Recently beautiful analytic proofs of the asymptotic of the Fourier coefficents of Mock-Theta functions have been obtained using a somewhat similar interplay [6].
} 
Our data for the 13 one-parameter models suggest further universal features. The purpose of this appendix is to describe some of these general features. Typical data for high degree look as is table A.1

The last nonzero entry is from the smooth genus 28 complete intersection curve 5 $(1,2,3,3)$ of degree 18 . By Castelnouvo's theory $\tilde{g}=28$ is the largest possible genus for degree 18. The degree one constraint parametrizes an $\mathbb{P}^{5}$. The moduli space $\mathcal{M}_{18}^{28}$ is a fibration of this $\mathbb{P}^{5}$ over a projectivization of the 15 parameters in the quadratic constraint. I.e. $\mathcal{M}_{18}^{28}$ is the total space of $\mathbb{P}^{5} \rightarrow \mathbb{P}^{14}$, with Euler number $\chi\left(\mathcal{M}_{d=18}^{g=28}\right)=5 \times 15=90$ and $n_{18}^{28}=(-1)^{5+14} 90=-90$.

As it can further be seen in table A.1, the numbers grow from genus $g=0$ to $g=3$ and fall thereafter. This feature might be related to the binomials in the description of the moduli of space as a singular fibration of the Jacobian $\mathrm{Jac}_{28}$ of the $g=28$ curve over $\mathcal{M}_{18}^{28}$. In this description the contribution of a $g=\tilde{g}-\delta$ curve comes from degenerating the genus 28 curve with $\delta$ nodes. As explained in [28] the contribution of the degenerate Jacobians can be expressed by the Euler numbers of relative Hilbert schemes $\mathcal{C}^{(n)}$ as

$$
n_{d}^{\tilde{g}-\delta}=(-1)^{\operatorname{dim}(\mathcal{M})+\delta} \sum_{p=0}^{\delta} b(\tilde{g}-p, \delta-p) \chi\left(\mathcal{C}^{(n)}\right),
$$

with $b(g, k)=\left(\begin{array}{c}2 g-2 \\ k\end{array}\right)$. A simple Gauss approximation of binomials fits the behaviour of the $n_{d}^{g}$ for large $d$ relatively well. We show this in Fig. 10 for the bi-cubic at degree 27. The numbers $n_{d}^{g}$ are exact and in contrast to (A.1) they count correctly all contribution from colliding nodes, all contributions from reducible curves as well as contributionsfrom smooth curves in the class $d$ with genus $\tilde{\tilde{g}}<\tilde{g}$.

Very important for the cancellations in the asymptotic behaviour of the DonaldsonThomas invariants is the occurrence of negative numbers. While it is clear that such contributions can arise if the dimensions of the D-brane moduli space is odd, we do not understand a priori the remarkable pattern with which these signs occur. The first occurrence of negative signs at $g_{n e g}(d)$ is graphed for the quintic and the bi-cubic in Fig. 11. The data suggest that $g_{n e g}(d)$ follows a parabola similar to the Castelnouvo bound. From the first occurrence of the negative sign the $n_{d}^{g}$ are alternating in sign for $g \ll \tilde{g}$. For $g \sim \tilde{g}$ the behaviour becomes more erratic. The Gauss approximation for the absolute values of the $n_{d}^{g}$ and the sign pattern is very characteristic of the degeneracies of microstates of a large black hole. In contrast the absolute value of the $n_{d}^{g}$ is falling and the signs are alternating with $(-1)^{g}$ starting at $g=0$ for small black holes as shown for example for the ST-model.

\footnotetext{
${ }^{5} \mathrm{~A}$ complete intersection curve $(1, n, 3,3)$ with degree $9 n$ has in general genus $\tilde{g}=\frac{1}{2}(1+3 n)(2+3 n)$.
} 


\begin{tabular}{|r|c|}
\hline genus & degree $=18$ \\
\hline 0 & 144519433563613558831955702896560953425168536 \\
1 & 491072999366775380563679351560645501635639768 \\
2 & 826174252151264912119312534610591771196950790 \\
3 & 866926806132431852753964702674971915498281822 \\
4 & 615435297199681525899637421881792737142210818 \\
5 & 306990865721034647278623907242165669760227036 \\
6 & 109595627988957833331561270319881002336580306 \\
7 & 28194037369451582477359532618813777554049181 \\
8 & 5218039400008253051676616144507889426439522 \\
9 & 688420182008315508949294448691625391986722 \\
10 & 63643238054805218781380099115461663133366 \\
11 & 4014173958414661941560901089814730394394 \\
12 & 166042973567223836846220100958626775040 \\
13 & 4251016225583560366557404369102516880 \\
14 & 61866623134961248577174813332459314 \\
15 & 451921104578426954609500841974284 \\
16 & 1376282769657332936819380514604 \\
17 & 1186440856873180536456549027 \\
18 & 2671678502308714457564208 \\
19 & -59940727111744696730418 \\
20 & 1071660810859451933436 \\
21 & -13279442359884883893 \\
22 & 101088966935254518, \\
23 & -372702765685392 \\
24 & 338860808028 \\
25 & 23305068 \\
26 & -120186 \\
27 & -5220 \\
28 & -90 \\
29 & 0 \\
\hline &
\end{tabular}

Table A.1: Gopakumar-Vafa invariants $n_{d}^{g}$ in the class $d=18$ for the complete intersection $X_{3,3}\left(1^{6}\right)$. 


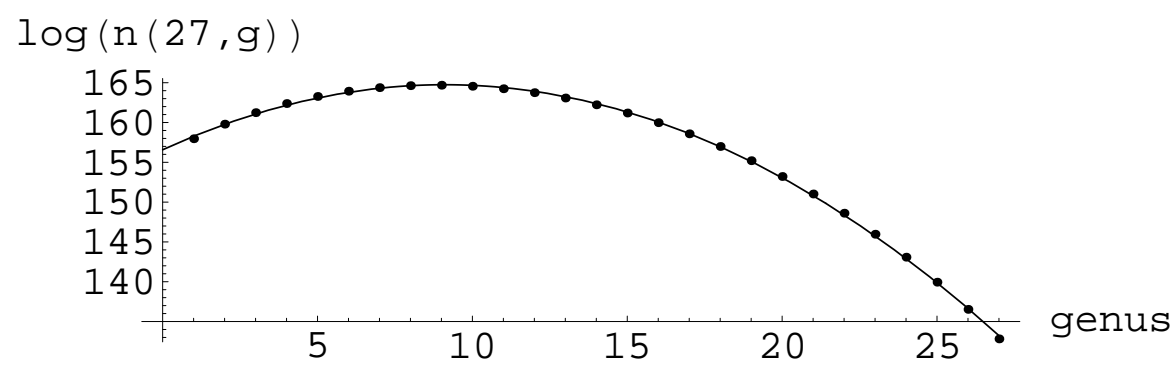

Figure 10: The binomials dominate the behaviour of large $d$ Gopakumar-Vafa invariants. For the degree 27 class on the bi-cubic we find $n_{27}^{g} \sim e^{167.747} e^{-0.0985(g-9.108)^{2}}$

\begin{tabular}{|rrrrrrrr|}
\hline$g$ & $d=1$ & 2 & 3 & 4 & 5 & 6 & 7 \\
\hline 0 & 2496 & 223752 & 38637504 & 9100224984 & 2557481027520 & 805628041231176 & $\ldots$ \\
1 & 0 & -492 & -1465984 & -1042943520 & -595277880960 & -316194812546140 & $\ldots$ \\
2 & 0 & -6 & 7488 & 50181180 & 72485905344 & 70378651228338 & $\ldots$ \\
3 & 0 & 0 & 0 & -902328 & -5359699200 & -10869145571844 & $\ldots$ \\
4 & 0 & 0 & 0 & 1164 & 228623232 & 1208179411278 & $\ldots$ \\
5 & 0 & 0 & 0 & 12 & -4527744 & -94913775180 & $\ldots$ \\
6 & 0 & 0 & 0 & 0 & 17472 & 4964693862 & $\ldots$ \\
7 & 0 & 0 & 0 & 0 & 0 & -152682820 & $\ldots$ \\
8 & 0 & 0 & 0 & 0 & 0 & 2051118 & $\ldots$ \\
9 & 0 & 0 & 0 & 0 & 0 & -2124 & $\ldots$ \\
10 & 0 & 0 & 0 & 0 & 0 & -22 & 605915136 \\
11 & 0 & 0 & 0 & 0 & 0 & 0 & -9419904 \\
12 & 0 & 0 & 0 & 0 & 0 & 0 & 32448 \\
\hline
\end{tabular}

A further remarkable fact is the very universal scaling for the maximal value $M(d)$ for $n_{d}^{g}$ for given $d$. This value behaves like

$$
M(d)=\exp \left((a+b d)^{4 / 3}\right)
$$

with very similar values for $a$ and $b$ for different one-parameter models, as shown for the quintic and the bi-cubic in Fig. 12 , 

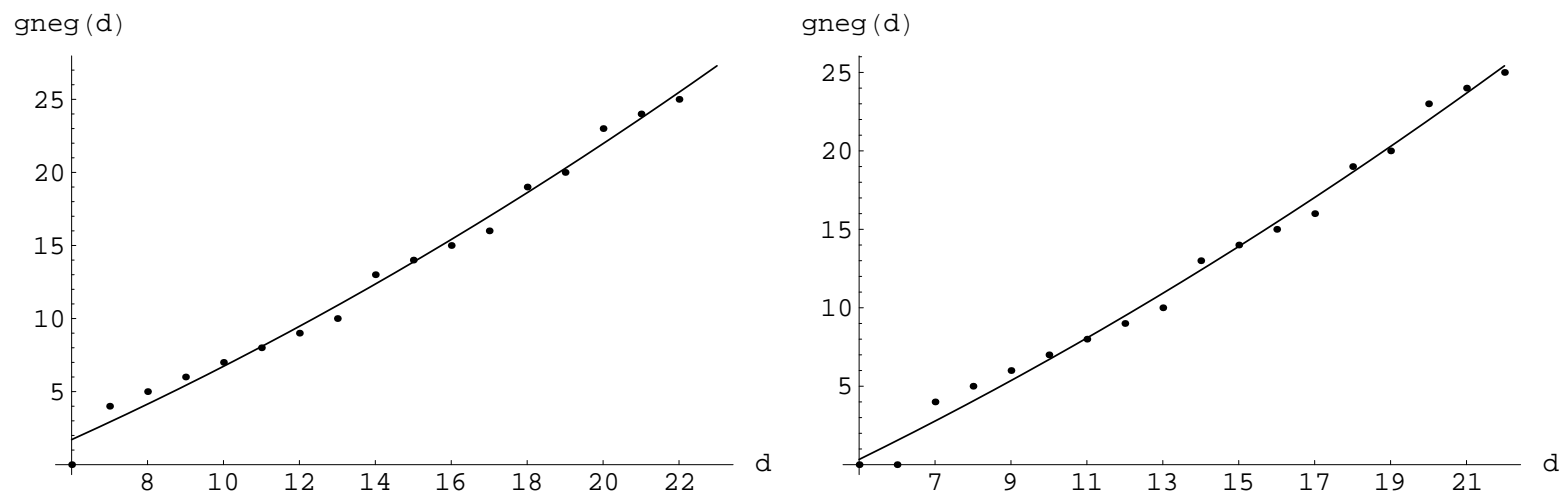

Figure 11: The first occurrence of negative $n_{d}^{g}$ for the quintic (on the right) and the bicubic (on the left). The fit is $m(d)=a+b d+c d^{2}$ with $a=-4.6, b=.94$ and $c=.019$ as well as $a=-5.2, b=1.0$ and $c=.017$ for these two, respectively.
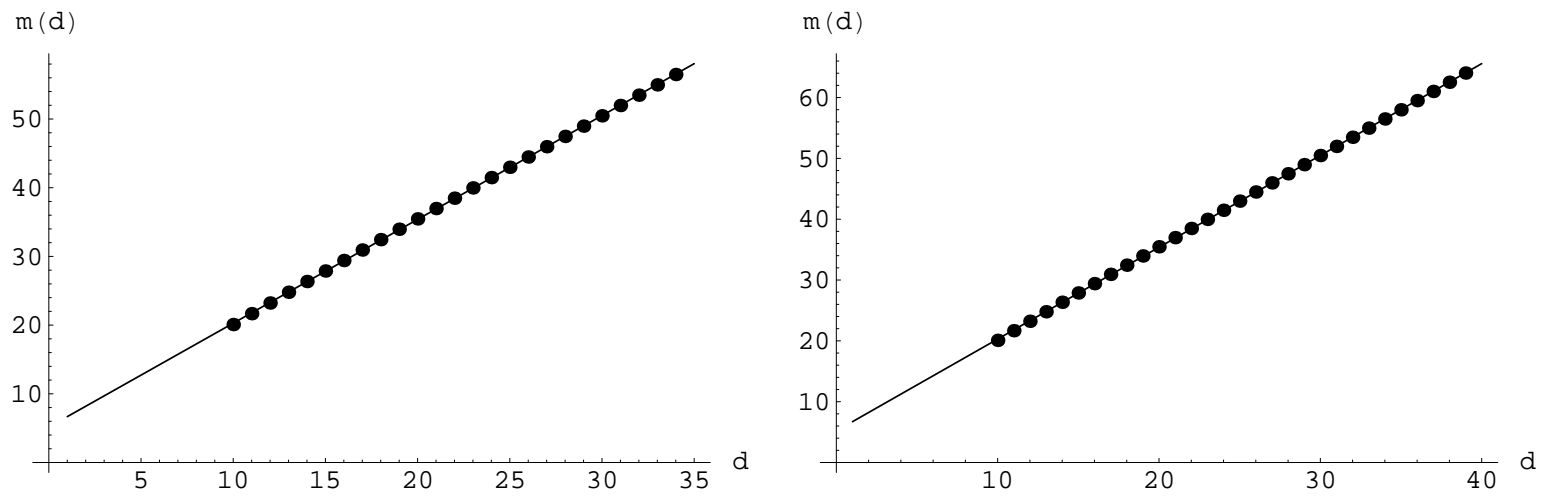

Figure 12: $m(d)=\log (M(d))^{3 / 4}$ for the quintic on the right and the bi-cubic on the left. $a=5.164$ and $b=1.511$ as well as $a=5.202$ and $b=1.509$ for the cases plotted. 


\section{References}

[1] M. Alishahiha, "On $R^{2}$ corrections for 5D black holes," arXiv:hep-th/0703099.

[2] I. Antoniadis, E. Gava, K. S. Narain and T. R. Taylor, "N=2 type II heterotic duality and higher derivative F terms," Nucl. Phys. B 455, 109 (1995) arXiv:hep-th/9507115].

[3] C. Bender and S. Orszag, Advanced mathematical methods for scientists and engineers, McGraw Hill, New York, 1978.

[4] M. Bershadsky, S. Cecotti, H. Ooguri and C. Vafa, "Kodaira-Spencer theory of gravity and exact results for quantum string amplitudes," Commun. Math. Phys. 165, 311 (1994) arXiv:hep-th/9309140.

[5] J. C. Breckenridge, R. C. Myers, A. W. Peet and C. Vafa, "D-branes and spinning black holes," Phys. Lett. B 391, 93 (1997) arXiv:hep-th/9602065.

[6] K. Bringmann and K. Ono, "The $f(q)$ mock theta function conjecture and partition ranks," Invent. math. 165, 243 (2006).

[7] G. L. Cardoso, B. de Wit and T. Mohaupt, "Corrections to macroscopic supersymmetric black-hole entropy," Phys. Lett. B 451, 309 (1999) arXiv:hep-th/9812082.

[8] A. Castro, J. L. Davis, P. Kraus and F. Larsen, "5D attractors with higher derivatives," arXiv:hep-th/0702072 "5D black holes and strings with higher derivatives," arXiv:hep-th/0703087.

[9] A. Dabholkar, F. Denef, G. W. Moore and B. Pioline, "Precision counting of small black holes," arXiv:hep-th/0507014.

[10] A. Dabholkar, N. Iizuka, A. Iqubal and M. Shigemori, "Precision microstate counting of small black rings," Phys. Rev. Lett. 96, 071601 (2006) arXiv:hep-th/0511120].

[11] J. R. David, G. Mandal and S. R. Wadia, "Microscopic formulation of black holes in string theory," Phys. Rept. 369, 549 (2002) [arXiv:hep-th/0203048].

[12] F. Denef and G. W. Moore, "Split states, entropy enigmas, holes and halos," arXiv:hep-th/0702146.

[13] D. E. Diaconescu and B. Florea, "Black hole entropy and Fourier-Mukai transform," arXiv:hep-th/0610068.

[14] R. Dijkgraaf, "Strings, matrices, and black holes," in Classical and quantum black holes, P. Fre et al. (eds.), IOP, 1999, p. 77-135. 
[15] R. Dijkgraaf, J. M. Maldacena, G. W. Moore and E. P. Verlinde, "A black hole farey tail," arXiv:hep-th/0005003.

[16] R. Dijkgraaf, G. W. Moore, E. P. Verlinde and H. L. Verlinde, "Elliptic genera of symmetric products and second quantized strings," Commun. Math. Phys. 185, 197 (1997) arXiv:hep-th/9608096].

[17] R. Dijkgraaf, E. P. Verlinde and H. L. Verlinde, "Counting dyons in N $=4$ string theory," Nucl. Phys. B 484, 543 (1997) arXiv:hep-th/9607026.

[18] S. Ferrara, J. A. Harvey, A. Strominger and C. Vafa, "Second quantized mirror symmetry," Phys. Lett. B 361, 59 (1995) [arXiv:hep-th/9505162].

[19] S. Ferrara and R. Kallosh, "Supersymmetry and Attractors," Phys. Rev. D 54, 1514 (1996) arXiv:hep-th/9602136].

[20] D. Gaiotto, A. Strominger and X. Yin, "New connections between 4D and 5D black holes," JHEP 0602, 024 (2006) arXiv:hep-th/0503217.

[21] R. Gopakumar and C. Vafa, "M-theory and topological strings. I\& II," arXiv:hep-th/9809187] and [arXiv:hep-th/9812127].

[22] T. W. Grimm, A. Klemm, M. Mariño and M. Weiss, "Direct integration of the topological string," arXiv:hep-th/0702187.

[23] M. Guica, L. Huang, W. Li and A. Strominger, " $\mathrm{R}^{* *} 2$ corrections for 5D black holes and rings," JHEP 0610, 036 (2006) [arXiv:hep-th/0505188].

[24] M. Guica and A. Strominger, "Wrapped M2/M5 duality," arXiv:hep-th/0701011.

[25] J. A. Harvey and G. W. Moore, "Algebras, BPS States, and Strings," Nucl. Phys. B 463, 315 (1996) arXiv:hep-th/9510182.

[26] M. x. Huang, A. Klemm and S. Quackenbush, "Topological string theory on compact Calabi-Yau: Modularity and boundary conditions," arXiv:hep-th/0612125.

[27] S. Kachru and C. Vafa, "Exact results for $\mathrm{N}=2$ compactifications of heterotic strings," Nucl. Phys. B 450, 69 (1995) arXiv:hep-th/9505105.

[28] S. Katz, A. Klemm and C. Vafa, "M-theory, topological strings and spinning black holes," Adv. Theor. Math. Phys. 3, 1445 (1999) arXiv:hep-th/9910181].

[29] T. Kawai, "String duality and modular forms," Phys. Lett. B 397, 51 (1997) [arXiv:hep-th/9607078]. 
[30] A. Klemm, M. Kreuzer, E. Riegler and E. Scheidegger, "Topological string amplitudes, complete intersection Calabi-Yau spaces and threshold corrections," JHEP 0505, 023 (2005) arXiv:hep-th/0410018.

[31] A. Klemm, W. Lerche and P. Mayr, "K3 Fibrations and heterotic type II string duality," Phys. Lett. B 357, 313 (1995) arXiv:hep-th/9506112.

[32] A. Klemm and M. Mariño, "Counting BPS states on the Enriques Calabi-Yau," arXiv:hep-th/0512227.

[33] M. Mariño, "Open string amplitudes and large order behavior in topological string theory," arXiv:hep-th/0612127.

[34] M. Mariño and G. W. Moore, "Counting higher genus curves in a Calabi-Yau manifold," Nucl. Phys. B 543, 592 (1999) arXiv:hep-th/9808131.

[35] D. Maulik, N. Nekrasov, A. Okounkov and R. Pandharipande, "Gromov-Witten theory and Donaldson-Thomas theory I," arXiv:math.AG/0312059.

[36] K. Oguiso, "On algebraic fiber space structures on a Calabi-Yau 3-fold," Internat. J. Math. 4, 439 (1993).

[37] H. Ooguri, A. Strominger and C. Vafa, "Black hole attractors and the topological string," Phys. Rev. D 70, 106007 (2004) arXiv:hep-th/0405146.

[38] S. H. Shenker, "The strength of nonperturbative effects in string theory," in O. Álvarez, E. Marinari and P. Windey (eds.), Random surfaces and quantum gravity, Plenum, New York, 1992.

[39] A. Strominger and C. Vafa, "Microscopic Origin of the Bekenstein-Hawking Entropy," Phys. Lett. B 379, 99 (1996) arXiv:hep-th/9601029].

[40] C. Vafa, "Black holes and Calabi-Yau threefolds," Adv. Theor. Math. Phys. 2, 207 (1998) arXiv:hep-th/9711067.

[41] R. M. Wald, "Black hole entropy in the Noether charge," Phys. Rev. D 48, 3427 (1993) [arXiv:gr-qc/9307038].

[42] S. Yamaguchi and S. T. Yau, "Topological string partition functions as polynomials," JHEP 0407, 047 (2004) arXiv:hep-th/0406078].

[43] http://uw.physics.wisc.edu/ strings/aklemm/blackholedata/ 